\title{
The cyclic sieving phenomenon on circular Dyck paths
}

\author{
Per Alexandersson \\ Department of Mathematics \\ Stockholm University \\ Stockholm, Sweden \\ per.w.alexandersson@gmail.com
}

\author{
Svante Linusson Samu Potka \\ Department of Mathematics \\ KTH Royal Institute of Technology \\ Stockholm, Sweden \\ \{linusson, potka\}@kth.se
}

Submitted: May 8, 2019; Accepted: Oct 1, 2019; Published: Oct 11, 2019

(C) The authors. Released under the CC BY-ND license (International 4.0).

\begin{abstract}
We give a $q$-enumeration of circular Dyck paths, which is a superset of the classical Dyck paths enumerated by the Catalan numbers. These objects have recently been studied by Alexandersson and Panova. Furthermore, we show that this $q$-analogue exhibits the cyclic sieving phenomenon under a natural action of the cyclic group. The enumeration and cyclic sieving is generalized to Möbius paths. We also discuss properties of a generalization of cyclic sieving, which we call subset cyclic sieving, and introduce the notion of Lyndon-like cyclic sieving that concerns special recursive properties of combinatorial objects exhibiting the cyclic sieving phenomenon.
\end{abstract}

Mathematics Subject Classifications: 05A15, 05A30, 05A19

\section{Introduction}

Unit interval graphs are in bijection with Dyck paths, and enumerated by the Catalan numbers, see e.g. Stanley's book on Catalan numbers [Sta15]. Recently, a natural generalization of these graphs was considered by Alexandersson and Panova [AP18] and also by Ellzey [Ell17a, Ell17b] in the study of Stanley chromatic symmetric functions. This generalization leads to an extension of Dyck paths to circular Dyck paths, see below for a precise definition.

The number of circular Dyck paths of size $n$ is given by the formula

$$
(n+2)\left(\begin{array}{c}
2 n-1 \\
n-1
\end{array}\right)-2^{2 n-1},
$$

and they are in bijection with pairs of Dyck paths of size $n$ with certain constraints, see A194460 in The On-Line Encyclopedia of Integer Sequences [Slo19]. Such pairs of Dyck 
paths have been studied in a different context by Baur and Mazorchuk [BM12], where they mentioned that Christian Krattenthaler previously has given a proof of (1) "via a lengthy combinatorial computation" starting from a recursion. Circular Dyck paths are described naturally by their area sequences, which extend the classical area sequences of Dyck paths, see e.g. the book by Haglund [Hag07].

The main results of this paper are listed below.

- We prove a $q$-analogue of (1) in Proposition 14. This also gives the first combinatorial proof of the fact that the number of circular Dyck paths is given by (1). In Section 3, we then generalize the $q$-analogue to circular Dyck paths with width w, obtaining

$$
\sum_{s \in \mathbb{Z}} \sum_{j=1}^{w} q^{s^{2}(w+2)+s(j+1)}\left(\left[\begin{array}{c}
2 n-1 \\
n-1-(w+2) s
\end{array}\right]_{q}-\left[\begin{array}{c}
2 n-1 \\
n+j+(w+2) s
\end{array}\right]_{q}\right)
$$

in Corollary 20. The $q$-analogue of (1) is the case $w=n$.

- In Theorem 22, we prove that circular Dyck paths of width $w$ together with (2) exhibit the cyclic sieving phenomenon (CSP) under a cyclic shift of the area sequence.

- In Section 5, we introduce and give a few examples of a phenomenon called subset cyclic sieving, where the values of a polynomial $f(q)$ at $n^{\text {th }}$ roots of unity give the number of elements in $Y \subseteq X$ fixed under a cyclic group action on $X$, and $f(1)$ is equal to the cardinality of $Y$.

- In Section 6, we prove a similar $q$-formula and instance of the CSP for paths embedded in a Möbius strip. In the process, we prove a new CSP instance for binary words of length $n$ under a twisted cyclic shift, with associated polynomial

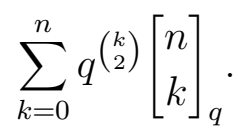

- In Section 7, we focus on families of CSP instances of a special type, parametrized by the size $n$ of the cyclic group. We ask the associated polynomials to fulfill the relation

$$
f_{n / m}(1)=f_{n}(\exp (2 \pi i / m)) \text { whenever } m \mid n \text {. }
$$

For example, this holds for the family of polynomials in (2) for each fixed $w \geqslant 1$. For natural reasons, we call such a sequence of CSP instances Lyndon-like, and we provide several more examples of this type.

Finally, we acknowledge that the On-line Encyclopedia of Integer Sequences, [Slo19], has been of great help in this project. This paper also benefited from experimentation with Sage [Dev19] and its combinatorics features developed by the Sage-Combinat community [com08]. 


\subsection{Brief background on the cyclic sieving phenomenon}

The cyclic sieving phenomenon (CSP) was introduced in 2004 by Reiner, Stanton and White [RSW04]. It generalizes Stembridge's $q=-1$ phenomenon [Ste94b, Ste94a, Ste96]. The definition consists of three ingredients: a finite set $X$, a cyclic group $C_{n}=\langle g\rangle$ of order $n$ acting on $X$, and a polynomial $f(q)$ with non-negative integer coefficients satisfying $f(1)=|X|$, for example a generating function for $X$. Let $\omega_{n}$ be a primitive $n^{\text {th }}$ root of unity, for example $e^{\frac{2 \pi i}{n}}$, and, as usual, let $[n]:=\{1, \ldots, n\}$.

Definition 1. The triple $\left(X, C_{n}, f(q)\right)$ exhibits the CSP if for every $k \in[n]$,

$$
[f(q)]_{q=\omega_{n}^{k}}=\left|\left\{x \in X: g^{k} \cdot x=x\right\}\right|,
$$

that is, $f(q)$ evaluated at the $k^{\text {th }}$ power of a primitive $n^{\text {th }}$ root of unity is the number of fixed points of $X$ under $g^{k}$.

Reiner, Stanton and White also gave an alternative, equivalent definition of the cyclic sieving phenomenon. The stabilizer-order of a $C$-orbit is the size of the stabilizer group of the elements of the orbit.

Proposition 2 ([RSW04]). The triple $(X, C, f(q))$ exhibits the CSP if $a_{\ell}$ defined by

$$
f(q) \equiv \sum_{\ell=0}^{n-1} a_{\ell} q^{\ell} \bmod \left(q^{n}-1\right)
$$

is the number of $C$-orbits on $X$ for which the stabilizer-order divides $\ell$.

By now, there is a multitude of CSP results. Below are some examples. For more, see for example the survey by Sagan [Sag11]. For the first one, we say that $g \in C$ with $|g|=n$ acts freely on $[N]$ if all of its cycles are of length $n$. A slight relaxation, we say that $g$ acts nearly freely on $[N]$ if it either acts freely or if all of its cycles have length $n$ except for one singleton. The cyclic group $C$ is said to act (nearly) freely on $[N]$ if it has a generator acting (nearly) freely on $[N]$. Finally, recall that $\left(\begin{array}{c}{[N]} \\ k\end{array}\right)$ and $\left(\left(\begin{array}{c}{[N]} \\ k\end{array}\right)\right)$ denote the sets of $k$-subsets and $k$-multisubsets of $[N]$, respectively.

Theorem 3 (Theorem 1.1, [RSW04]). Suppose $C$ is a cyclic group acting nearly freely on $[N]$. Then

$$
\left(\left(\left(\begin{array}{c}
{[N]} \\
k
\end{array}\right)\right), C,\left[\begin{array}{c}
N+k-1 \\
k
\end{array}\right]_{q}\right) \text { and }\left(\left(\begin{array}{c}
{[N]} \\
k
\end{array}\right), C,\left[\begin{array}{c}
N] \\
k
\end{array}\right]_{q}\right)
$$

exhibit the CSP.

Rhoades [Rho10] proved a CSP result for rectangular standard Young tableaux. For a more geometric version, for example in terms non-crossing matchings in the two-row case, see the work by Petersen, Pylyavskyy and Rhoades [PPR08]. 
Theorem 4 (Theorem 1.3, [Rho10]). If $\lambda=\left(n^{m}\right)$, then

$$
\left(\operatorname{SYT}(\lambda),\langle\partial\rangle, f^{\lambda}(q)\right)
$$

exhibits the CSP, where $\operatorname{SYT}(\lambda)$ is the set of standard Young tableaux of the shape $\lambda,\langle\partial\rangle$ is the cyclic group generated by the jeu-de-taquin promotion operator, and $f^{\lambda}(q)$ is the natural q-analogue of the hook-length formula.

In the two-row case $\lambda=(n, n)$, note that there is a bijection between $\operatorname{SYT}(\lambda)$ and Dyck paths of size $n$. Pechenik [Pec14] proved that the small Schröder paths also exhibit the CSP.

Another result specializing to lattice paths is the following. The major index of a word $w$ of length $n$ is the sum of the indices $i \in[n-1]$ such that $w_{i}>w_{i+1}$. A pair $(i, j)$ is an inversion of $w$ if $i<j$ but $w_{i}>w_{j}$, and $\operatorname{inv}(w)$ is the number of inversions in $w$.

Theorem 5 (A reformulation of [RSW04, Prop. 4.4]). Let $X_{n}(\mu)$ be the set of words of length $n$ and content $\mu$, that is, each word has $\mu_{i}$ entries equal to $i$. Let the cyclic group $C_{n}$ act on $X$ by cyclic shift, and let

$$
f_{n}(\mu ; q):=\left[\begin{array}{l}
n \\
\mu
\end{array}\right]_{q}=\sum_{w \in X_{n}(\mu)} q^{\operatorname{maj}(w)}=\sum_{w \in X_{n}(\mu)} q^{\operatorname{inv}(w)} .
$$

Then $\left(X_{n}, C_{n}, f_{n}(\mu ; q)\right)$ exhibits the CSP.

In the case $\mu=\left(\mu_{1}, \mu_{2}\right)$ with $\mu_{1}+\mu_{2}=n, X_{n}(\mu)$ is in an obvious bijection with, for example, lattice paths starting at $(0,0)$ and with steps from $\{(0,1),(1,0)\}$. Ahlbach and Swanson [AS18] have proved a refinement of this Theorem 5 .

\subsection{Background on $q$-analogues}

In the previous examples we saw how q-analogues appear in the context of the cyclic sieving phenomenon. We will also encounter them in this paper and hence introduce them here. The starting point is the definition $[n]_{q}:=\frac{1-q^{n}}{1-q}=1+q+\cdots+q^{n-1}$, which is motivated by the observation

$$
\lim _{q \rightarrow 1} \frac{1-q^{n}}{1-q}=n .
$$

Then it is natural to define the q-factorial

$$
\begin{aligned}
{[n]_{q} ! } & :=[1]_{q} \cdot[2]_{q} \cdots[n-1]_{q} \cdot[n]_{q}=\frac{1-q}{1-q} \cdot \frac{1-q^{2}}{1-q} \cdots \frac{1-q^{n-1}}{1-q} \cdot \frac{1-q^{n}}{1-q} \\
& =1 \cdot(1+q) \cdots\left(1+q+\cdots+q^{n-2}\right) \cdot\left(1+q+\cdots+q^{n-1}\right) .
\end{aligned}
$$

While $n$ ! counts the number of permutations on $[n]$, it is well-known (see, for example, $\left[\right.$ Sta11]) that $[n]_{q} !=\sum_{\sigma \in S_{n}} q^{\operatorname{inv}(\sigma)}$ 
Having defined $q$-factorials, the next natural step is to define $q$-binomial coefficients (also called Gaussian binomial coefficients, Gaussian coefficients and Gaussian polynomials) by

$$
\left[\begin{array}{l}
n \\
k
\end{array}\right]_{q}:=\frac{[n]_{q} !}{[n-k]_{q} ![k]_{q} !} \text { for } 0 \leqslant k \leqslant n
$$

and letting $\left[\begin{array}{l}n \\ k\end{array}\right]_{q}:=0$ otherwise. One combinatorial interpretation of the $q$-binomial coefficient is that it counts the number of $k$-dimensional subspaces of the $n$-dimensional vector space over the $q$-element field, see [Sta11] for the details.

Many identities for binomial coefficients have their counterparts for $q$-binomial coefficients. For example, we have the symmetry

$$
\left[\begin{array}{l}
n \\
k
\end{array}\right]_{q}=\left[\begin{array}{c}
n \\
n-k
\end{array}\right]_{q}
$$

and the $q$-Pascal identities

$$
\left[\begin{array}{l}
n \\
k
\end{array}\right]_{q}=q^{k}\left[\begin{array}{c}
n-1 \\
k
\end{array}\right]_{q}+\left[\begin{array}{l}
n-1 \\
k-1
\end{array}\right]_{q} \text { and }\left[\begin{array}{l}
n \\
k
\end{array}\right]_{q}=\left[\begin{array}{c}
n-1 \\
k
\end{array}\right]_{q}+q^{n-k}\left[\begin{array}{l}
n-1 \\
k-1
\end{array}\right]_{q} .
$$

A useful tool for proving CSP results is the $q$-Lucas theorem below. We shall make use of the following notation. Given $a \in \mathbb{Z}$ and $d \in \mathbb{N}$, let $\{a\}_{d}$ denote the remainder of $a$ $\bmod d$, so that $a=d\lfloor a / d\rfloor+\{a\}_{d}$.

Lemma 6 (See, for example, [Sag92]). We have that

$$
\left[\begin{array}{l}
n \\
k
\end{array}\right]_{q} \equiv\left(\begin{array}{l}
\lfloor n / d\rfloor \\
\lfloor k / d\rfloor
\end{array}\right)\left[\begin{array}{l}
\{n\}_{d} \\
\{k\}_{d}
\end{array}\right]_{q} \bmod \Phi_{d}
$$

where $\Phi_{d}$ is the $d^{\text {th }}$ cyclotomic polynomial.

In particular, Lemma 6 implies that with $q$ a primitive $d^{\text {th }}$ root of unity,

$$
\left[\begin{array}{l}
n \\
k
\end{array}\right]_{q}=\left(\begin{array}{l}
\lfloor n / d\rfloor \\
\lfloor k / d\rfloor
\end{array}\right)\left[\begin{array}{l}
\{n\}_{d} \\
\{k\}_{d}
\end{array}\right]_{q},
$$

a fact we use extensively in later sections.

The following two well-known results due to MacMahon are also related to our work.

Lemma 7 (See [And76, Thm. 3.6]). Let BW $(k, m)$ denote the set of binary words of length $k+m$ with $k 1 s$. Then

$$
\sum_{\mathbf{b} \in \mathrm{BW}(k, m)} q^{\operatorname{maj}(\mathbf{b})}=\left[\begin{array}{c}
k+m \\
k
\end{array}\right]_{q}
$$


Proposition 8 (See e.g. the lemma on p. 255 in [FH85]). The major index of binary words corresponding to Dyck paths generates the classical Carlitz q-analogue of the Catalan numbers:

$$
\sum_{D \in \operatorname{DP}(n)} q^{\operatorname{maj}(D)}=\frac{1}{[n+1]_{q}}\left[\begin{array}{c}
2 n \\
n
\end{array}\right]_{q} .
$$

When evaluating the right-hand side at $e^{2 \pi i k / n}$, we obtain non-negative integers which count fixed points under promotion. That action can be described either by bijecting to $2 \times n$ SYT, or by considering $2 \pi / n$ rotations of perfect matchings in a $2 n$-gon. The special case of Theorem 4 mentioned in the previous section is a refinement of this, using rotations of $\pi / n$ instead.

\section{Enumeration of circular Dyck paths}

A Dyck path of size $n$ is a sequence of north steps $(0,1)$ and east steps $(1,0)$ starting from $(0,0)$ and ending at $(n, n)$ that lies weakly above the line $y=x$. It may be described via its area sequence. For example, the path $(0,1,2,3,2,2)$ corresponds to

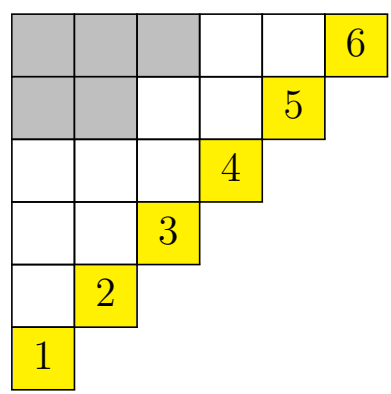

where the area sequence specifies the number of white squares in each row, from bottom to top. The number of Dyck paths of size $n$ is given by the $n^{\text {th }}$ Catalan number, $\frac{1}{n+1}\left(\begin{array}{c}2 n \\ n\end{array}\right)$.

A circular Dyck path of size $n$ is specified via an area sequence $a_{1}, \ldots, a_{n}$ satisfying

- $0 \leqslant a_{i} \leqslant n-1$ for $1 \leqslant i \leqslant n$,

- $a_{i+1} \leqslant a_{i}+1$ for $1 \leqslant i \leqslant n$,

where the index is taken mod $n$ in the second condition. This set is denoted $\operatorname{CDP}(n)$. The subset of paths with $a_{1}=0$ corresponds to classical Dyck paths, $\mathrm{DP}(n)$. Circular Dyck paths can also be illustrated as diagrams. 
Example 9. For example, $\mathbf{a}=(3,4,2,3,2,3)$ is illustrated as

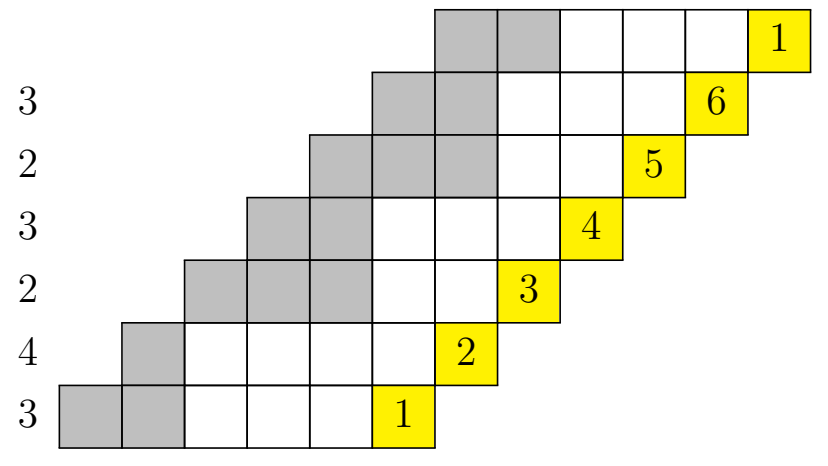

where the top row is a repetition of the first row to illustrate the cyclic nature of the path.

It is often convenient to describe circular Dyck paths as paths along the border of the white squares, see Figure 1. For this to be uniquely defined, one has to fix a starting point $\mathbf{x}=\left(x_{0}, 0\right), 1 \leqslant x_{0} \leqslant n$. We denote such a path by $(\mathbf{x}, \mathbf{b})$, where $\mathbf{b}=\left(b_{1}, \ldots, b_{2 n}\right) \in$ $\{0,1\}^{2 n}$ is a binary sequence with $n$ os and $1 \mathrm{~s}$, respectively. Here 0 corresponds to an east step and 1 to a north step, with $b_{2 n}=1$, that is, the last step is up.

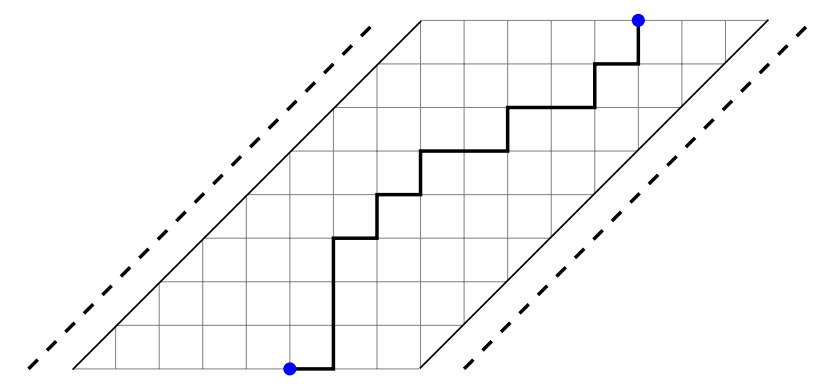

Figure 1: Example of the bijection with lattice paths, with $n=8$. The path in the middle, starting at $(6,0)$ and with binary word 0111010100100101 , corresponds to the area sequence $\mathbf{a}=(2,3,4,4,4,3,2,2)$. The area sequence is determined by the number of (whole) squares to the right of the path in each row. The two dashed diagonals are never touched by a lattice path corresponding to an area sequence.

The condition $b_{2 n}=1$ is needed to make the starting point well defined. Note that one may not have $x_{0}=n+1$, since the last step is up, it would mean passing the illegal point $(2 n+1, n-1)$.

We also study circular Dyck paths with a width different from the height, which are defined in an analogous manner. A circular Dyck path of height $n$ and width $w$ is specified via an area sequence such that $a_{1}, \ldots, a_{n}$ satisfy

- $0 \leqslant a_{i} \leqslant w-1$ for $1 \leqslant i \leqslant n$,

- $a_{i+1} \leqslant a_{i}+1$ for $1 \leqslant i \leqslant n$, 
where the index is taken mod $n$ in the second condition. This set is denoted $\operatorname{CDP}(n, w)$. Equivalently we can think of the elements in $\operatorname{CDP}(n, w)$ as $(\mathbf{x}, b)$, where $\mathbf{x}=\left(x_{0}, 0\right)$, $1 \leqslant x_{0} \leqslant w$, is the starting point and $\mathbf{b}=\left(b_{1}, \ldots, b_{n+w}\right) \in\{0,1\}^{n+w}$ is a binary sequence with $n$ 1s and $w 0$ s such that the corresponding path stays between the diagonals $y=x$ and $y=x-(w+2)$, and $b_{n+w}=1$.

There is a natural $C_{n}$-action on $\operatorname{CDP}(n, w)$, where the generator shifts the area sequence cyclically by one step to the right. We let $\alpha$ denote such a cyclic shift.

\subsection{Bijection with tuples of Dyck paths}

There is a bijection between circular Dyck paths and pairs of Dyck paths with certain peak conditions. A peak in a Dyck path is a north step followed by an east step. In the area sequence, this corresponds to $a_{i+i} \leqslant a_{i}$ or to the last number $a_{n}$. The height of the first peak of a Dyck path $Q$, denoted $h_{f}(Q)$, is the number of north steps before the first east step, or equivalently, $a_{i}+1$ for the smallest $i$ such that $a_{i+i} \leqslant a_{i}$ (or $a_{n}+1$ if no such $i$ exists). Similarly we define the height of the last peak, $h_{l}(Q)$, as the number of ending east steps after the last north step, or equivalently $a_{n}+1$.

For the reflected Dyck path $P$ in the following lemma, think of the roles of east and north steps as being reversed.

Lemma 10 ([AP18, Lem. 5]). Circular Dyck paths of size $n$ are in bijection with pairs $(P, Q)$ of ordinary Dyck paths of size $n$, such that

$$
h_{f}(P)+h_{l}(Q) \geqslant n \quad \text { and } \quad h_{l}(P)+h_{f}(Q) \geqslant n .
$$

The bijection in the previous lemma is illustrated in Figure 2.

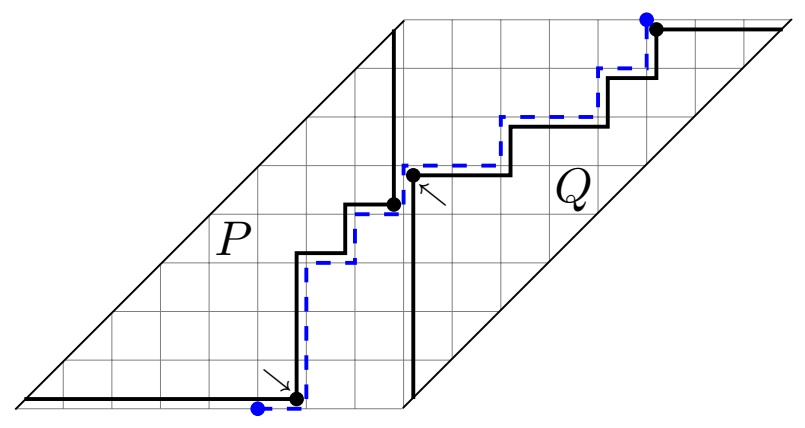

Figure 2: The bijection with pairs of Dyck paths. The circular area sequence of the circular Dyck path in the figure is $(2,3,4,4,4,3,2,2)$. The first and last peaks have been marked, and arrows point the first peak in each path.

Lemma 11. The set $\operatorname{CDP}(k n, n)$ is in bijection with $2 k$-tuples of Dyck paths $\left(P_{1}, \ldots, P_{2 k}\right)$ such that

$$
h_{l}\left(P_{j}\right)+h_{f}\left(P_{j+1}\right) \geqslant n, \text { for } 1 \leqslant j<2 k, \quad \text { and } \quad h_{l}\left(P_{2 k}\right)+h_{f}\left(P_{1}\right) \geqslant n .
$$

Proof. This follows from simply extending the idea in Lemma 10, as shown in Figure 3. 


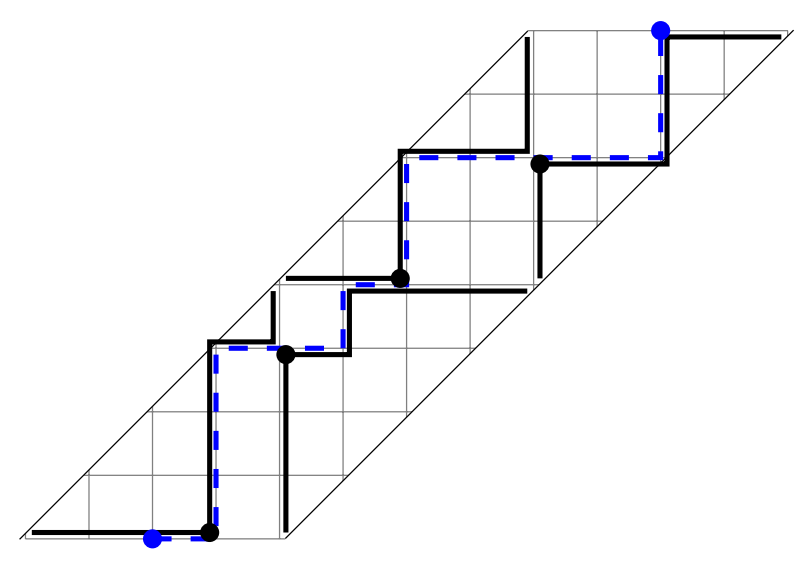

Figure 3: Here we have a path (dashed) from $\operatorname{CDP}(8,4)$, which is mapped to a 4-tuple of Dyck paths of size 4 (solid). The first peak has been marked in each Dyck path.

\subsection{Circular Möbius paths}

It is natural to ask what happens if we have a single Dyck path $P$ of size $n$, such that $(P, P)$ satisfies the peak condition in Lemma 10. It is straightforward to show that such Dyck paths are in bijection with $(\mathbf{x}, \mathbf{b})$ in $\operatorname{CDP}(n)$ such that $\mathbf{b}=\left(b_{1}, \ldots, b_{2 n}\right)$ fulfills the relation

$$
b_{i}=1-b_{n+i} \quad \text { for all } \quad i \in[n] .
$$

Note that, in particular, $b_{n}=0$ as we always have $b_{2 n}=1$. The starting point $\mathbf{x}=\left(x_{0}, 0\right)$ is uniquely determined by $\mathbf{b}$ since $b_{n}=0$ must correspond to an east step that ends on the vertical line $x=n+1$. From this it is easy to see that all possible $\mathbf{b}$ correspond to exactly one path. We let $\operatorname{CMP}(n) \subseteq \operatorname{CDP}(n)$ denote this set, and refer to such paths as circular Möbius paths, see Figure 4 for an example.
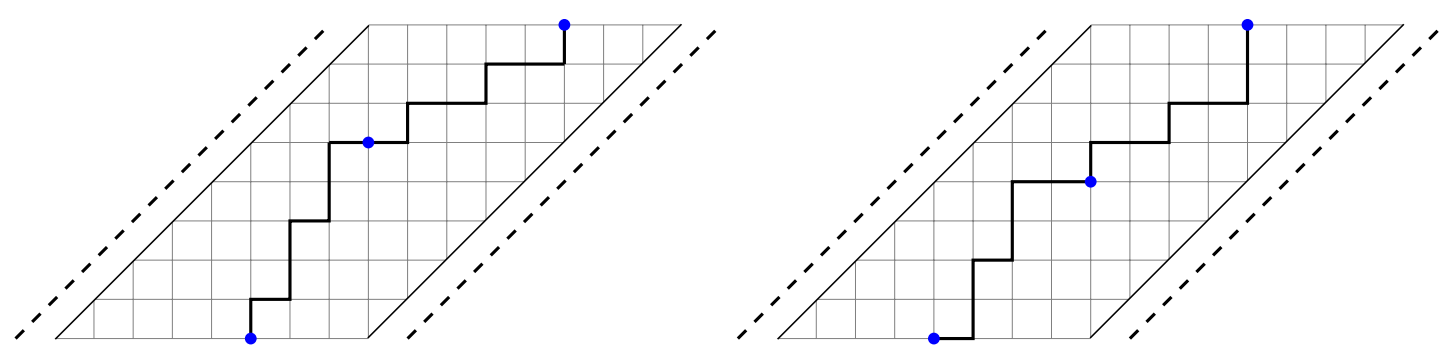

Figure 4: Two Möbius paths in $\operatorname{CMP}(8)$. Note that each path is the concatenation of two smaller paths, the second part being a reflection of the first. Hence the name "Möbius".

Lemma 12. We have that $|\operatorname{CMP}(n)|=2^{n-1}$.

Proof. As noted above, a path in $\operatorname{CMP}(n)$ is determined uniquely by the first $n-1$ steps in $\mathbf{b}$. 
Problem 1. $\operatorname{DP}(n)$ is in bijection with permutations of $[n]$ avoiding a pattern $\tau$ of length $3, \operatorname{Av}_{n}(\tau)$. There are also several pairs $\left(\tau_{1}, \tau_{2}\right)$ of patterns of length 3 such that $\left|\operatorname{Av}_{n}\left(\tau_{1}, \tau_{2}\right)\right|=2^{n-1}$. At the same time, $\operatorname{CMP}(n) \subseteq \mathrm{DP}(n)$ has $2^{n-1}$ elements. It could be interesting to find a natural bijection between $\operatorname{DP}(n)$ and $\operatorname{Av}_{n}(\tau)$, for some $\tau$, such that restricted to $\operatorname{CMP}(n)$ it becomes a bijection to $\operatorname{Av}_{n}\left(\tau, \tau^{\prime}\right)$, where $\tau^{\prime}$ is some other pattern of length 3 .

\section{$2.3 \quad$ A $q$-analogue}

As mentioned, circular Dyck paths and NE-lattice paths in general correspond to pairs $(\mathbf{x}, \mathbf{b})$ of a starting point $\mathbf{x}$ and a binary word $\mathbf{b}$ where $b_{i}=1$ if the $i^{\text {th }}$ step is a north step, and $b_{i}=0$ otherwise. For example, the path in Figure 2 gives the word 0100100101011101.

The major index of a circular Dyck path a (corresponding to $(\mathbf{x}, \mathbf{b})$ ) is defined as the major index of the binary word $\mathbf{b}$. Recall that the major index of a binary word $w$ of length $n$ is the sum of all $i \in[n-1]$ such that $w_{i}>w_{i+1}$.

Define the following $q$-analogue of circular Dyck paths:

$$
|\operatorname{CDP}(n, w)|_{q}:=\sum_{(\mathbf{x}, \mathbf{b}) \in \operatorname{CDP}(n, w)} q^{\operatorname{maj}(\mathbf{b})}
$$

We end this section by proving a $q$-analogue of $(1)$ in the introduction, that enumerates $\operatorname{CDP}(n)$. The proof mimics the ideas of [FH85, p. 255]. In the next section, we extend the method and do the same for $\operatorname{CDP}(n, w)$.

We begin the proof with a lemma generalizing $q$-ballot numbers.

Lemma 13. Summing over all north-east paths $\mathbf{b}$ starting in $(x, 0)$, ending in $(i, j)$, and never touching the $x=y$ diagonal, and with $i \geqslant j, x \geqslant 0$, we get

$$
\sum_{\mathbf{b}} q^{\operatorname{maj}(\mathbf{b})}=\left[\begin{array}{c}
i+j-x \\
j
\end{array}\right]_{q}-q^{x}\left[\begin{array}{c}
i+j-x \\
j-x
\end{array}\right]_{q} .
$$

Proof. We proceed by induction over $x$. For $x=0$ it is clearly true since then there are no paths. The maj-count of all north-east paths from $(x, 0)$ to $(i, j)$ is $\left[\begin{array}{c}i+j-x \\ j\end{array}\right]_{q}$, and we will now count and subtract the paths that touch the diagonal $x=y$. The idea for this proof comes from [FH85, p. 255], where they construct a major-index preserving bijection between sets of lattice paths.

In this proof we define the depth of a path to measure how far beyond the diagonal $x=y$ the path goes, or, more formally, to be the largest value of $s-r$ for any point $(r, s)$ on the path. We now define a bijection $\varphi$ that maps a path $\mathbf{b}$ ending in $(i, j)$ with depth $d \geqslant 0$ to a path ending in $(i+1, j-1)$ with depth $d-1$.

Let $(r, s)$ be the first point of maximal depth on the path. Since $x>0,(r, s)$ is not the starting point. If it is the point directly after the starting point, we must have $r=s=1$. Otherwise, the last two steps reaching $(r, s)$ are north steps. The map $\varphi$ is defined by switching the north step just before $(r, s)$ to an east step, that is, relabeling $b_{r+s-x}$ from 1 to 0 . In $\varphi(\mathbf{b})$ the position $(r, s-1)$ will then be the last point of maximal depth and 
it is thus easy to find it and define $\varphi^{-1}$. The corner in position $(r, s)$ has been replaced with a corner in $(r, s-1)$, unless $r=s=1$. In any case, $\operatorname{maj}(\mathbf{b})=\operatorname{maj}(\varphi(\mathbf{b}))+1$. Thus $\varphi$ is a bijection between the paths from $(x, 0)$ to $(i, j)$ that touch the diagonal $x=y$ and paths from $(x, 0)$ to $(i+1, j-1)$ that touch the diagonal $x=y+1$, with a shift of $q^{1}$. By induction, the maj-count of paths from $(x, 0)$ to $(i+1, j-1)$ that do not touch the diagonal $x=y+1$ is

$$
\left[\begin{array}{c}
i+j-1-(x-1) \\
j-1
\end{array}\right]_{q}-q^{x-1}\left[\begin{array}{c}
i+j-1-(x-1) \\
j-1-(x-1)
\end{array}\right]_{q},
$$

and thus the maj-count of those touching the diagonal $x=y+1$ is

$$
q^{x-1}\left[\begin{array}{c}
i+j-1-(x-1) \\
j-1-(x-1)
\end{array}\right]_{q}=q^{x-1}\left[\begin{array}{c}
i+j-x \\
j-x
\end{array}\right]_{q} .
$$

Using $\varphi$ we thus get that the maj-count of the paths from $(x, 0)$ to $(i, j)$ that touch the diagonal $x=y$ is $q^{x}\left[\begin{array}{c}i+j-x \\ j-x\end{array}\right]_{q}$, which gives the formula claimed in the lemma.

Proposition 14. For any $n \geqslant 1$,

$$
|\mathrm{CDP}(n)|_{q}=n\left[\begin{array}{c}
2 n-1 \\
n-1
\end{array}\right]_{q}-\sum_{j=1}^{n} q^{j}\left[\begin{array}{c}
2 n-1 \\
n+j
\end{array}\right]_{q}-\sum_{j=1}^{n}\left[\begin{array}{c}
2 n-1 \\
j-2
\end{array}\right]_{q} .
$$

Proof. For each possible starting point $(x, 0), 1 \leqslant x \leqslant n$, the maj-count of all paths to $(x+n, n-1)$ (remember that the last step of $\mathbf{b}$ is a north step) is $\left[\begin{array}{c}2 n-1 \\ n-1\end{array}\right]_{q}$. This gives the first term. We will now subtract the paths that touch the surrounding diagonals. Note that no path can touch both diagonals. By Lemma 13 the maj-count of paths touching the diagonal $x=y$ is $\sum_{x=1}^{n-1} q^{j}\left[\begin{array}{c}2 n-1 \\ n-1-x\end{array}\right]_{q}$, which gives the first sum.

For paths touching the diagonal $x=y+n+2$ we can use the bijection defined dually to $\varphi$ in the proof of Lemma 13. That is, we change an east step to a north step for the first corner being diagonally furthest to the right. This time there clearly is no shift in the maj of the path and we get the second sum.

\section{A formula for the $q$-analogue for circular Dyck paths}

The goal of this section is to express $|\operatorname{CDP}(n, w)|_{q}$ as a sum of $q$-binomial coefficients. To achieve this, we need to consider the major index generating function for arbitrary north-east lattice paths starting at the origin and with some constraints, which will be used in an inclusion-exclusion argument.

A diagonal is a set of lattice points of the form $\{\mathbf{x}+k(1,1): k \in \mathbb{Z}\}$ for some $\mathbf{x} \in \mathbb{Z}^{2}$. It is clear that a diagonal is uniquely specified by any point on the diagonal. For a lattice point $\mathbf{y}$ in the non-negative quadrant, let

$$
H\left(\mathbf{y} ; d_{1}, d_{2}, \ldots, d_{\ell}\right), \quad d_{i} \in \mathbb{Z}
$$


denote the $q$-enumeration (using major index) of north-east lattice paths $L$ from $(0,0)$ to $\mathbf{y}$, such that $L$ includes points from each of the $\ell$ diagonals specified by the points

$$
d_{i}(1,0) \quad i=1,2, \ldots, \ell,
$$

in this order. In other words, there must be points $p_{1}, \ldots, p_{\ell}$ on $L$ visited in this order, such that $p_{i}$ is on the diagonal specified by $d_{i}$. Note that by definition

$$
H\left(\mathbf{y} ; 0, d_{1}, d_{2}, \ldots, d_{\ell}\right)=H\left(\mathbf{y} ; d_{1}, d_{2}, \ldots, d_{\ell}\right)
$$

since the starting point $(0,0)$ is on the diagonal specified by 0 . Abusing notation, we henceforth let the diagonal $d_{i}$ be the unique diagonal specified by $d_{i}$.

We say that the configuration $\left(\mathbf{y} ; d_{1}, \ldots, d_{\ell}\right)$ is alternating if any of the four conditions below is fulfilled:

1. $0 \geqslant d_{1}<d_{2}>d_{3}<d_{4}>\cdots>d_{\ell}$ and $\mathbf{y}$ is to the right of diagonal $d_{\ell}$,

2. $0 \geqslant d_{1}<d_{2}>d_{3}<d_{4}>\cdots<d_{\ell}$ and $\mathbf{y}$ is to the left of diagonal $d_{\ell}$,

3. $0 \leqslant d_{1}>d_{2}<d_{3}>d_{4}<\cdots<d_{\ell}$ and $\mathbf{y}$ is to the left of diagonal $d_{\ell}$ or

4. $0 \leqslant d_{1}>d_{2}<d_{3}>d_{4}<\cdots>d_{\ell}$ and $\mathbf{y}$ is to the right of diagonal $d_{\ell}$.

By convention, if $\ell=0$, the configuration is considered to be alternating as well. Note that $H\left(\mathbf{y} ; d_{1}\right)=H(\mathbf{y})$ if $(0,0)$ and $\mathbf{y}$ are on different sides of $d_{1}$. Note also more generally that given a non-alternating subsequence $d_{k}>d_{k+1}>d_{k+2}$ or $d_{k}<d_{k+1}<d_{k+2}$ of diagonals, $H(\mathbf{y} ; \mathbf{d})=H\left(\mathbf{y} ; \mathbf{d}^{\prime}\right)$ where $\mathbf{d}^{\prime}$ denotes $\mathbf{d}$ with $d_{k+1}$ removed.

Let nw denote the vector $(-1,1)$ and recall that we identify east steps with 0 and north steps with 1.

Lemma 15. Suppose that $\left(\mathbf{y} ; d_{1}, \ldots, d_{\ell}\right)$ is alternating and $\ell \geqslant 1$. Then the generating function $H\left(\mathbf{y} ; d_{1}, \ldots, d_{\ell}\right)$ is equal to

$$
\left\{\begin{array}{l}
H\left(\mathbf{y}-\mathbf{n w} ; d_{1}+1, d_{2}+2, d_{3}+2, \ldots, d_{\ell}+2\right) \times q \text { if } d_{1}<0 \\
H\left(\mathbf{y}+\mathbf{n w} ; d_{1}-1, d_{2}-2, d_{3}-2, \ldots, d_{\ell}-2\right) \quad \text { otherwise }
\end{array}\right.
$$

Furthermore, both new configurations above are alternating as well.

Proof. The proof is a more complicated version of the proof of Lemma 13. We have two different cases to consider: $d_{1}<0$ and $d_{1}>0$. Let us start with the former. In all cases, we let $(r, s)$ be the first point maximizing the depth $\operatorname{sgn}\left(d_{1}\right)(x-y)$ among points $(x, y) \in L$ before $L$ meets the diagonal $d_{2}$. In other words, $(r, s)$ is the first point furthest away from $d_{1}$ on the side opposite of $(0,0)$, or on $d_{1}$ if $L$ does not cross $d_{1}$.

Case $\boldsymbol{d}_{\mathbf{1}}<\mathbf{0}$ : Suppose $L$ is a path counted by $H\left(\mathbf{y} ; d_{1}, \ldots, d_{\ell}\right)$. A north step 1 has to precede $(r, s)$, while an east step 0 has to follow it.

Let $\phi$ be the map replacing the north step 1 preceding $(r, s)$ with an east step. Note that $\phi$ is similar to $\varphi$ in the proof of Lemma 13 but now only the points of $L$ before it meets $d_{2}$ are considered. 


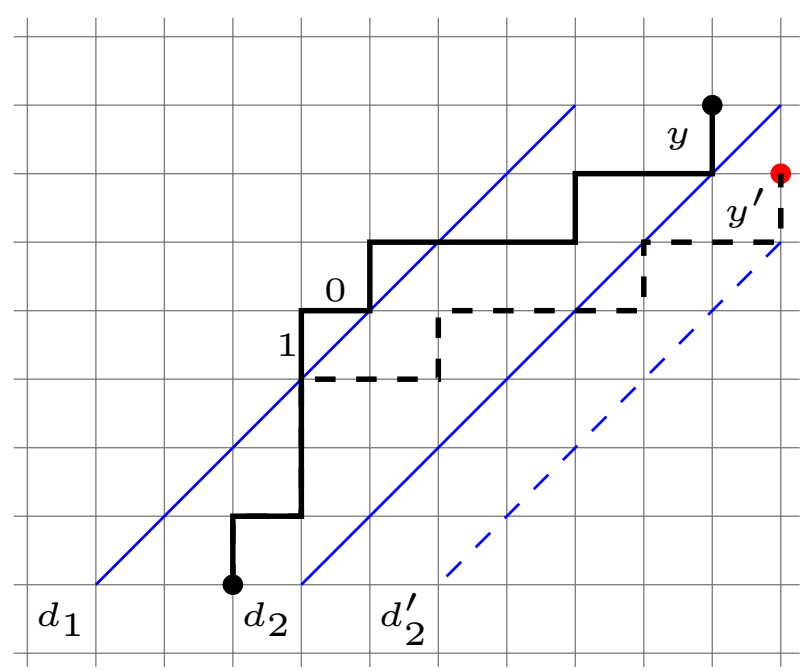

Figure 5: The map $\phi$. In the figure, $d_{1}=-2$ and $d_{2}=1$. The path $L$ is shown as a solid line and $\phi(L)$ is dashed. We have the new endpoint $y^{\prime}=y-\mathbf{n w}$ and the shifted diagonal $d_{2}^{\prime}=3$. Note that $L$ touches $d_{2}$ in the same manner as $\phi(L)$ touches $d_{2}^{\prime}$.

Now, $\operatorname{maj}(\phi(L))=\operatorname{maj}(L)-1$, the new endpoint is given by $\mathbf{y}-\mathbf{n w}$ and $\phi(L)$ hits the shifted diagonals $d_{1}+1, d_{2}+2, d_{3}+2, \ldots, d_{\ell}+2$, see Figure 5 .

It is evident that $\phi$ is invertible. The inverse is given by replacing the east step 0 following the last deepest point (that is, maximizing $y-x$ ) after touching the diagonal $d_{1}+1$ and before the diagonal $d_{2}+2$ with a north step 1 .

Case $\boldsymbol{d}_{\mathbf{1}}>\mathbf{0}$ : In this case, construct a bijection $\psi$ by replacing the east step 0 preceding $(r, s)$ with a north step 1 .

Note that this does not affect the major index and that $\psi(L)$ ends at $\mathbf{y}+\mathbf{n w}$. Furthermore, $\psi(L)$ intersects all diagonals $d_{1}-1$ as well as $d_{2}-2, d_{3}-2$, and so on. As before, it is straightforward to show that $\psi$ has an inverse. This proves the second case of $(9)$.

Corollary 16. If $\left(\mathbf{y} ; d_{1}, \ldots, d_{\ell}\right)$ is alternating, then $H\left(\mathbf{y} ; d_{1}, \ldots, d_{\ell}\right)$ is equal to

$$
H\left(\mathbf{y}+d_{1} \mathbf{n w} ; d_{2}-2 d_{1}, d_{3}-2 d_{1}, \ldots, d_{\ell}-2 d_{1}\right) \times\left\{\begin{array}{l}
q^{-d_{1}} \text { if } d_{1}<0 \\
1 \text { otherwise }
\end{array}\right.
$$

Proof. Apply Lemma 15 repeatedly.

We shall now focus on generating functions of lattice paths that touch two diagonals at least $\ell$ times in an alternating fashion. Let $\langle a, b\rangle_{\ell}$ denote the alternating list $(a, b, a, b, \ldots)$ of length $\ell$.

Corollary 17. Suppose $\delta>\gamma>0$. Then for all $j=0, \ldots,\lfloor\ell / 2\rfloor$, we have the identities

$$
H\left(\mathbf{y} ;\langle\gamma, \gamma-\delta\rangle_{\ell}\right)=q^{j^{2} \delta+j \gamma} H\left(\mathbf{y}-j \delta \mathbf{n w} ;\langle\gamma+2 j \delta, \gamma-\delta+2 j \delta\rangle_{\ell-2 j}\right)
$$


and

$$
H\left(\mathbf{y} ;\langle\gamma-\delta, \gamma\rangle_{\ell}\right)=q^{j^{2} \delta-j \gamma} H\left(\mathbf{y}+j \delta \mathbf{n w} ;\langle\gamma-\delta-2 j \delta, \gamma-2 j \delta\rangle_{\ell-2 j}\right)
$$

Proof. The first identity is proved by applying the recursion in (10) two times, and using induction over $j$. The first application on the expression

$$
H\left(\mathbf{y}-j \delta \mathbf{n w} ;\langle\gamma+2 j \delta, \gamma-\delta+2 j \delta\rangle_{\ell-2 j}\right)
$$

gives

$$
H\left(\mathbf{y}+(j \delta+\gamma) \mathbf{n w} ;\langle-\gamma-\delta-2 j \delta,-\gamma-2 j \delta\rangle_{\ell-2 j-1}\right) .
$$

The second application of the recursion gives

$$
q^{\gamma+\delta+2 j \delta} H\left(\mathbf{y}-(j \delta+\delta) \mathbf{n w} ;\langle\gamma+2 \delta+2 j \delta, \gamma+\delta+2 j \delta\rangle_{\ell-2 j-2}\right) .
$$

Finally, we observe that

$$
\left(j^{2} \delta+j \gamma\right)+(\gamma+\delta+2 j \delta)=(j+1)^{2} \delta+(j+1) \gamma
$$

so the result now follows via induction over $j$. The second identity is proved in a similar fashion.

Lemma 18. Suppose $\delta>\gamma>0$ and $\mathbf{y}=(n, n-1)$. We then have the identities

$$
\begin{aligned}
H\left(\mathbf{y} ;\langle\gamma, \gamma-\delta\rangle_{2 \ell}\right) & =q^{\ell^{2} \delta+\ell \gamma}\left[\begin{array}{c}
2 n-1 \\
n-1-\delta \ell
\end{array}\right]_{q}, \quad \ell \geqslant 0 \\
H\left(\mathbf{y} ;\langle\gamma, \gamma-\delta\rangle_{2 \ell+1}\right) & =q^{\ell^{2} \delta+\ell \gamma}\left[\begin{array}{c}
2 n-1 \\
n-1+\gamma+\delta \ell
\end{array}\right]_{q}, \quad \ell \geqslant 0, \\
H\left(\mathbf{y} ;\langle\gamma-\delta, \gamma\rangle_{2 \ell}\right) & =q^{\ell^{2} \delta-\ell \gamma}\left[\begin{array}{c}
2 n-1 \\
n-1+\delta \ell
\end{array}\right]_{q}, \quad \ell \geqslant 0, \\
H\left(\mathbf{y} ;\langle\gamma-\delta, \gamma\rangle_{2 \ell-1}\right) & =q^{\ell^{2} \delta-\ell \gamma}\left[\begin{array}{c}
2 n-1 \\
n-1+\gamma-\delta \ell
\end{array}\right]_{q}, \quad \ell \geqslant 1 .
\end{aligned}
$$

Proof. Note that in all cases we deal with north-east lattice paths of length $2 n-1$ with exactly $n$ east steps, which we interpret as binary words of length $2 n-1$ with exactly $n$ 0s. From this observation, it is straightforward to see that Corollary 17 together with Lemma 7 implies the first and third identity.

To prove (12), note that Corollary 17 and (9) gives

$$
\begin{aligned}
H\left(\mathbf{y} ;\langle\gamma, \gamma-\delta\rangle_{2 \ell+1}\right) & =q^{\ell^{2} \delta+\ell \gamma} H\left(\mathbf{y}-(\ell \delta) \mathbf{n w} ;\langle\gamma+2 \ell \delta\rangle_{1}\right) \\
& =q^{\ell^{2} \delta+\ell \gamma} H\left(\mathbf{y}+(\gamma+\ell \delta) \mathbf{n w} ;\langle\cdot\rangle_{0}\right) \\
& =q^{\ell^{2} \delta+\ell \gamma}\left[\begin{array}{c}
2 n-1 \\
(n-1)+(\gamma+\ell \delta)
\end{array}\right]_{q}
\end{aligned}
$$


Finally, the last identity follows from the fact that

$$
\begin{aligned}
& H\left(\mathbf{y} ;\langle\gamma-\delta, \gamma\rangle_{2 \ell-1}\right) \\
& =q^{(\ell-1)^{2} \delta-\gamma(\ell-1)} H\left(\mathbf{y}+(-\delta+\ell \delta) \mathbf{n w} ;\langle\gamma-\delta-2(\ell-1) \delta\rangle_{1}\right) \\
& =q^{(\ell-1)^{2} \delta-\gamma(\ell-1)-\gamma+\delta+2(\ell-1) \delta} H\left(\mathbf{y}+(-\delta+\ell \delta+\gamma-\delta-2 \ell \delta+2 \delta) \mathbf{n w} ;\langle\cdot\rangle_{0}\right) \\
& =q^{\ell^{2} \delta-\gamma \ell} H\left(\mathbf{y}+(\gamma-\ell \delta) \mathbf{n w} ;\langle\cdot\rangle_{0}\right) \\
& =q^{\ell^{2} \delta-\gamma \ell}\left[\begin{array}{c}
2 n-1 \\
n-1+\gamma-\ell \delta
\end{array}\right]_{q}
\end{aligned}
$$

This finishes the proof of the identities.

Definition 19. Fix an integer $w \geqslant 0$ and $j \in\{1, \ldots, w\}$ and let the diagonals through $(0,0)$ and $(w+2,0)$ be referred to as the left and the right diagonal, respectively.

Let $L_{j}(n, w, \ell)$ be the $q$-enumeration of north-east paths $L$ from

$$
(w+1-j, 0) \text { to }(n+w+1-j, n-1)
$$

with the property that there are $\ell$ points on the path $L, p_{1}, p_{2}, \ldots, p_{\ell}$, appearing in this order from the start, such that the odd-indexed $p_{i}$ lie on the left diagonal, and the even-indexed $p_{i}$ are points on the right diagonal. Similarly, define $R_{j}(n, w, \ell)$ to be the $q$-enumeration of north-east paths in (15) such that there are $\ell$ points $p_{1}, p_{2}, \ldots, p_{\ell}$ on the path with the even-indexed $p_{i}$ being on the left diagonal, and the odd-indexed $p_{i}$ being points on the right diagonal.

Let $\delta:=w+2$ and $\mathbf{y}=(n, n-1)$. From Definition 19 , it is straightforward to see that the generating functions $L_{j}(n, w, \ell)$ and $R_{j}(n, w, \ell)$ are equal to the generating functions in Lemma 18. Unraveling the definitions, we have that

$$
\begin{array}{rlrl}
L_{j}(n, w, 2 \ell) & =H\left(\mathbf{y} ;\langle j+1-\delta, j+1\rangle_{2 \ell}\right) & & =q^{\ell^{2} \delta-\ell(j+1)}\left[\begin{array}{c}
2 n-1 \\
n-1+\delta \ell
\end{array}\right]_{q} \\
L_{j}(n, w, 2 \ell+1) & =H\left(\mathbf{y} ;\langle j+1-\delta, j+1\rangle_{2 \ell+1}\right) & & =q^{\ell^{2} \delta-\ell(j+1)}\left[\begin{array}{c}
2 n-1 \\
n+j-\delta \ell
\end{array}\right]_{q} \\
R_{j}(n, w, 2 \ell) & =H\left(\mathbf{y} ;\langle j+1, j+1-\delta\rangle_{2 \ell}\right) & & =q^{\ell^{2} \delta+\ell(j+1)}\left[\begin{array}{c}
2 n-1 \\
n-1-\delta \ell
\end{array}\right]_{q} \\
R_{j}(n, w, 2 \ell-1) & =H\left(\mathbf{y} ;\langle j+1, j+1-\delta\rangle_{2 \ell-1}\right) & =q^{\ell^{2} \delta+\ell(j+1)}\left[\begin{array}{c}
2 n-1 \\
n+j+\delta \ell
\end{array}\right]_{q}
\end{array}
$$

Corollary 20. We have the q-enumeration

$$
\left.\operatorname{CDP}(n, w)\right|_{q}=\sum_{s \in \mathbb{Z}} \sum_{j=1}^{w} q^{s^{2} \delta+s(j+1)}\left(\left[\begin{array}{c}
2 n-1 \\
n-1-\delta s
\end{array}\right]_{q}-\left[\begin{array}{c}
2 n-1 \\
n+j+\delta s
\end{array}\right]_{q}\right),
$$

where $\delta=w+2$. In particular, when $w \geqslant n$, we have

$$
|\operatorname{CDP}(n, w)|_{q}=w\left[\begin{array}{c}
2 n-1 \\
n-1
\end{array}\right]_{q}-\sum_{j=1}^{w} q^{j}\left[\begin{array}{c}
2 n-1 \\
n+j
\end{array}\right]_{q}-\sum_{j=1}^{w}\left[\begin{array}{c}
2 n-1 \\
n+j-(w+2)
\end{array}\right]_{q} .
$$


Proof. We have that $\operatorname{CDP}(n, w)$ are certain north-east lattice paths avoiding the two diagonals through $(0,0)$ and $(\delta, 0)$. To find the maj-count of these paths, we use an inclusion-exclusion argument. Not taking the restrictions imposed by the diagonals into account, the maj-count is given by

$$
\sum_{j=1}^{w}\left[\begin{array}{c}
2 n-1 \\
n-1
\end{array}\right]_{q}=w\left[\begin{array}{c}
2 n-1 \\
n-1
\end{array}\right]_{q} .
$$

The paths counted by $L_{j}(n, w, 1)$ and $R_{j}(n, w, 1)$ for $j \in[w]$ enumerate all forbidden paths. However, we cannot simply subtract both as there are paths counted by both of these expressions, namely $L_{j}(n, w, 2)$ and $R_{j}(n, w, 2)$, and so on. Combining Definition 19 and the enumeration in Lemma 18 then gives the expression in (16).

Note that, in particular, $L_{j}(n, w, 2)=R_{j}(n, w, 2)=0$ whenever $w \geqslant n$ (since a path cannot hit both forbidden diagonals in this case) so we get the less complicated expression in (17). Letting $w=n$ in (17) gives (7).

Lemma 21. We have the identity

$$
\begin{aligned}
|\operatorname{CDP}(n, w)| & =(w+2) \sum_{t \in \mathbb{Z}}\left(\begin{array}{c}
2 n-1 \\
n+(w+2) t
\end{array}\right)-\sum_{t \in \mathbb{Z}}\left(\begin{array}{c}
2 n-1 \\
n+t
\end{array}\right) \\
& =(w+2) \sum_{t \in \mathbb{Z}}\left(\begin{array}{c}
2 n-1 \\
n+(w+2) t
\end{array}\right)-2^{2 n-1} .
\end{aligned}
$$

Proof. This is a straightforward consequence of (16), by letting $q=1$ and then adding and subtracting the case $j=0$ and $j=w+1$ to the inner sum.

We note that the $q=1$ case of (16) follows easily from a result by Mohanty [Moh79, Thm. 2], where the proof is also done via a reflection argument together with inclusionexclusion. However, his approach is not compatible with our use of major index.

\section{The cyclic sieving phenomenon under shifting}

This section contains the proof of our main result, Theorem 22 stated below.

Theorem 22. Let $\alpha$ act on $\operatorname{CDP}(n, w)$ by cyclically shifting the area sequence one step. Then the triple

$$
\left(\operatorname{CDP}(n, w),\langle\alpha\rangle,|\operatorname{CDP}(n, w)|_{q}\right)
$$

is a CSP-triple.

The proof consists of first counting the number of fixed points under cyclic shift by $k$ steps, which is done in Lemma 23. Then, in Proposition 25, we show that the $q$-analogue $|\operatorname{CDP}(n, w)|_{q}$ evaluates to it at $q=e^{2 \pi i k / n}$.

Let $\operatorname{CDP}_{k}(n, w)$ be the subset of area sequences in $\operatorname{CDP}(n, w)$ that is fixed by a cyclic shift of $k$ steps. 
Lemma 23. For $n \geqslant k \geqslant 1$, let $d:=\operatorname{gcd}(n, k)$, then

$$
\left|\mathrm{CDP}_{k}(n, w)\right|=|\mathrm{CDP}(d, w)|
$$

Proof. This is straightforward to prove.

Let $\delta:=w+2$. We have that $|\operatorname{CDP}(n, w)|_{q}$ is equal to

$$
\sum_{s \in \mathbb{Z}} \sum_{j=1}^{w} q^{s^{2} \delta+s(j+1)}\left(\left[\begin{array}{c}
2 n-1 \\
n+\delta s
\end{array}\right]_{q}-\left[\begin{array}{c}
2 n-1 \\
n+j+\delta s
\end{array}\right]_{q}\right) .
$$

We need to evaluate this at powers of $\exp (2 \pi i / n)$. All such powers are of the form $\exp (2 \pi i \ell / m)$, where $m \mid n$ and $\operatorname{gcd}(\ell, m)=1$. The goal is to show that if $n=m d$, then

$$
|\operatorname{CDP}(n, w)|_{q=\exp (2 \pi i \ell / m)}=|\operatorname{CDP}(d, w)| .
$$

This identity is trivial whenever $m=1$, so we assume that $m \geqslant 2$. Recall that $\{d\}_{m}$ denotes the (non-negative) remainder of $d$ when divided by $m$. The $q$-Lucas theorem implies that whenever $q=\exp (2 \pi i \ell / m)$ for $n=m d, \operatorname{gcd}(\ell, m)=1$, we have that $|\operatorname{CDP}(n, w)|_{q}$ is equal to

$$
\sum_{s \in \mathbb{Z}} \sum_{j=1}^{w} q^{s^{2} \delta+s(j+1)}\left(\left(\begin{array}{c}
2 d-1 \\
d+\left\lfloor\frac{\delta s}{m}\right\rfloor
\end{array}\right)\left[\begin{array}{l}
m-1 \\
\{\delta s\}_{m}
\end{array}\right]_{q}-\left(\begin{array}{c}
2 d-1 \\
d+\left\lfloor\frac{\delta s+j}{m}\right\rfloor
\end{array}\right)\left[\begin{array}{c}
m-1 \\
\{j+\delta s\}_{m}
\end{array}\right]_{q}\right) .
$$

Introduce

$$
\begin{aligned}
& A(s, j)=q^{s^{2} \delta+s(j+1)}\left(\begin{array}{c}
2 d-1 \\
d+\left\lfloor\frac{\delta s}{m}\right\rfloor
\end{array}\right)\left[\begin{array}{l}
m-1 \\
\{\delta s\}_{m}
\end{array}\right]_{q} \\
& B(s, j)=-q^{s^{2} \delta+s(j+1)}\left(\begin{array}{c}
2 d-1 \\
d+\left\lfloor\frac{\delta s+j}{m}\right\rfloor
\end{array}\right)\left[\begin{array}{c}
m-1 \\
\{j+\delta s\}_{m}
\end{array}\right]_{q}
\end{aligned}
$$

which also implicitly depend on $\delta$ and $m$.

The following lemma is needed for the proof of Proposition 25.

Lemma 24. We have the following identities:

(I) $A(s, j)=-A(-s, w-j)$, for $j \neq w, \delta s \not \equiv_{m} 0$.

(II) $B(s, j)=-B(-s-1, w-j)$, for $j \neq w, j+1+\delta s \not \equiv_{m} 0$.

(III) $A(s, w)=-B(s-1, w)$ for $\delta s \not \equiv_{m} 0,1$.

(IV) $B(s-1, w)+\sum_{j=1}^{w} A(-s, j)=-\left(\begin{array}{c}2 d-1 \\ d+\frac{\delta s}{m}-1\end{array}\right)$ whenever $\delta s \equiv_{m} 0$ and $s \not \equiv_{m} 0$.

(V) $A(s, j)=\left(\begin{array}{c}2 d-1 \\ d+\frac{\delta s}{m}\end{array}\right)$ whenever $s \equiv_{m} 0$. 
(VI) $A(s, w)=-\left(\begin{array}{c}2 d-1 \\ d+\frac{\delta s-1}{m}\end{array}\right)$ whenever $\delta s \equiv_{m} 1$.

(VII) $B(s, j)=-\left(\begin{array}{c}2 d-1 \\ d+\frac{\delta+j+1}{m}-1\end{array}\right)$ whenever $j+1+\delta s \equiv_{m} 0$ and $j<w$.

(VIII) $B(s-1, w)=\left(\begin{array}{c}2 d-1 \\ d+\frac{\delta s}{m}-1\end{array}\right)$ whenever $s \equiv_{m} 0$.

(IX) $B(s-1, w)=-\left(\begin{array}{c}2 d-1 \\ d+\frac{\delta s-1}{m}-1\end{array}\right)$ whenever $\delta s \equiv_{m} 1$.

Furthermore, over all combinations of $s \in \mathbb{Z}$ and $j=1,2, \ldots, w$, the above cases cover each term in (21) exactly once.

Proof. Case I: For $q=\exp (2 \pi i \ell / m)$, we want to show $A(s, j)=-A(-s, w-j)$, whenever $1 \leqslant j<w$ and $\delta s \not_{m} 0$. We must prove that

$$
q^{s^{2} \delta+s(j+1)}\left(\begin{array}{c}
2 d-1 \\
d+\lfloor\delta s / m\rfloor
\end{array}\right)\left[\begin{array}{l}
m-1 \\
\{\delta s\}_{m}
\end{array}\right]_{q}=-q^{s^{2} \delta-s(w-j+1)}\left(\begin{array}{c}
2 d-1 \\
d+\lfloor-\delta s / m\rfloor
\end{array}\right)\left[\begin{array}{c}
m-1 \\
\{-\delta s\}_{m}
\end{array}\right]_{q}
$$

Let us first assume that $s>0$ and let $r:=(\delta s \bmod m)$, so that $0<r<m$. We must show that

$$
\begin{aligned}
q^{s \delta}\left(\begin{array}{c}
2 d-1 \\
d+\lfloor\delta s / m\rfloor
\end{array}\right)\left[\begin{array}{c}
m-1 \\
r
\end{array}\right]_{q} & =-\left(\begin{array}{c}
2 d-1 \\
d-1-\lfloor\delta s / m\rfloor
\end{array}\right)\left[\begin{array}{l}
m-1 \\
m-r
\end{array}\right]_{q} \\
q^{r}\left(\begin{array}{c}
2 d-1 \\
d+\lfloor\delta s / m\rfloor
\end{array}\right) \frac{1-q^{m-r}}{1-q^{m}}\left[\begin{array}{c}
m \\
r
\end{array}\right]_{q} & =-\left(\begin{array}{c}
2 d-1 \\
d+\lfloor\delta s / m\rfloor
\end{array}\right) \frac{1-q^{m-(m-r)}}{1-q^{m}}\left[\begin{array}{c}
m \\
m-r
\end{array}\right]_{q} \\
q^{r}\left(1-q^{m-r}\right) & =q^{r}-1
\end{aligned}
$$

which is true. The case $s<0$ is treated in a similar manner.

Case II: For $q=\exp (2 \pi i \ell / m)$, we want to show $B(s, j)=-B(-s-1, w-j)$ whenever $1 \leqslant j<w$ and $j+\delta s+1 \not \equiv_{m} 0$. We must prove that

$$
\begin{aligned}
& q^{s^{2} \delta+s(j+1)}\left(\begin{array}{c}
2 d-1 \\
d+\left\lfloor\frac{j+\delta s}{m}\right\rfloor
\end{array}\right)\left[\begin{array}{l}
m-1 \\
\{\delta s\}_{m}
\end{array}\right]_{q} \\
= & -q^{(-s-1)^{2} \delta+(-s-1)(w-j+1)}\left(\begin{array}{c}
2 d-1 \\
d+\left\lfloor\frac{w-j+\delta(-s-1)}{m}\right\rfloor
\end{array}\right)\left[\begin{array}{c}
m-1 \\
\{w-j+\delta(-s-1)\}_{m}
\end{array}\right]_{q} \\
= & -q^{s(j+\delta s+1)+j+\delta s+1}\left(\begin{array}{c}
2 d-1 \\
d+\left\lfloor\frac{-2-j-\delta s}{m}\right\rfloor
\end{array}\right)\left[\begin{array}{c}
m-1 \\
\{-2-j-\delta s\}_{m}
\end{array}\right]_{q} \\
= & -q^{s(j+\delta s+1)+j+\delta s+1}\left(\begin{array}{c}
2 d-1 \\
d-1-\left\lfloor\frac{j+\delta s}{m}\right\rfloor
\end{array}\right)\left[\begin{array}{c}
m-1 \\
\{-2-j-\delta s\}_{m}
\end{array}\right]_{q} .
\end{aligned}
$$

Now, let $j+\delta s \equiv_{m} r, 0<r<m$. Then, we need to show

$$
\begin{aligned}
& q^{s(r+1)}\left(\begin{array}{c}
2 d-1 \\
d+\left\lfloor\frac{j+\delta s}{m}\right\rfloor
\end{array}\right)\left[\begin{array}{c}
m-1 \\
r
\end{array}\right]_{q} \\
= & -q^{s(r+1)+r+1}\left(\begin{array}{c}
2 d-1 \\
d-1-\left\lfloor\frac{j+\delta s}{m}\right\rfloor
\end{array}\right)\left[\begin{array}{c}
m-1 \\
r+1
\end{array}\right]_{q} .
\end{aligned}
$$


This follows from that

$$
q^{r+1}\left[\begin{array}{c}
m-1 \\
r+1
\end{array}\right]_{q}=q^{r+1}\left[\begin{array}{c}
m-1 \\
r
\end{array}\right]_{q} \frac{1-q^{m-r-1}}{1-q^{r+1}}=-\left[\begin{array}{c}
m-1 \\
r
\end{array}\right]_{q} .
$$

Case III: Let $r \equiv_{m} \delta(s+1)$ with $0 \leqslant r<m$. We want to prove that $A(s+1, w)=$ $-B(s, w)$ under the condition that $r \notin\{0,1\}$, which implies that $s \neq-1$.

This amounts to proving

$$
\begin{gathered}
q^{(s+1)^{2} \delta+(s+1)(\delta-1)}\left(\begin{array}{c}
2 d-1 \\
d+\lfloor\delta(s+1) / m\rfloor
\end{array}\right)\left[\begin{array}{c}
m-1 \\
\{\delta(s+1)\}_{m}
\end{array}\right]_{q}= \\
q^{s^{2} \delta+s(\delta-1)}\left(\begin{array}{c}
2 d-1 \\
d+\lfloor(\delta(s+1)-2) / m\rfloor
\end{array}\right)\left[\begin{array}{c}
m-1 \\
\{\delta(s+1)-2\}_{m}
\end{array}\right]_{q}
\end{gathered}
$$

Since the binomials are equal under our conditions, it is enough to show that

$$
\begin{aligned}
q^{2 r}\left[\begin{array}{c}
m-1 \\
r
\end{array}\right]_{q} & =q\left[\begin{array}{c}
m-1 \\
r-2
\end{array}\right]_{q} \\
q^{2 r} \frac{[m-r]_{q}}{[r]_{q}}\left[\begin{array}{c}
m-1 \\
r-1
\end{array}\right]_{q} & =q \frac{[r-1]_{q}}{[m-r+1]_{q}}\left[\begin{array}{c}
m-1 \\
r-1
\end{array}\right]_{q} \\
q^{2 r} \frac{1-q^{-r}}{1-q^{r}} & =q \frac{1-q^{r-1}}{1-q^{1-r}}
\end{aligned}
$$

and it is easy to verify that these are equal.

Case IV: We need to prove that

$$
B(s-1, w)+\sum_{1 \leqslant j \leqslant w} A(-s, j)=-\left(\begin{array}{c}
2 d-1 \\
d+\frac{\delta s}{m}-1
\end{array}\right)
$$

whenever $\delta s \equiv_{m} 0, s \not_{m} 0$ and $q=\exp (2 \pi i \ell / m)$. Note that $m \geqslant 2$ in this case. Inserting the definitions, we need to evaluate

$$
\begin{aligned}
& -q^{(s-1)^{2} \delta+(s-1)(w+1)}\left(\begin{array}{c}
2 d-1 \\
d+\lfloor(w+\delta(s-1)) / m
\end{array}\right)\left[\begin{array}{c}
m-1 \\
w+\delta(s-1)\}_{m}
\end{array}\right]_{q}+ \\
& \left(\sum_{j=1}^{w} q^{s^{2} \delta-s(j+1)}\right)\left(\begin{array}{c}
2 d-1 \\
d+\lfloor-\delta s / m\rfloor
\end{array}\right)\left[\begin{array}{c}
m-1 \\
\{-\delta s\}_{m}
\end{array}\right]_{q}
\end{aligned}
$$

After some simplifications, this is equal to

$$
-q^{1-s}\left(\begin{array}{c}
2 d-1 \\
d+\lfloor(\delta s-2) / m\rfloor
\end{array}\right)\left[\begin{array}{c}
m-1 \\
\{\delta s-2\}_{m}
\end{array}\right]_{q}+q^{-s}\left(\sum_{j=1}^{w} q^{-s j}\right)\left(\begin{array}{c}
2 d-1 \\
d+\frac{\delta s}{m}-1
\end{array}\right)
$$

which becomes

$$
-q^{1-s}\left(\begin{array}{c}
2 d-1 \\
d+\frac{\delta s}{m}-1
\end{array}\right)\left[\begin{array}{c}
m-1 \\
m-2
\end{array}\right]_{q}+q^{-s}\left(\sum_{j=1}^{w} q^{-s j}\right)\left(\begin{array}{c}
2 d-1 \\
d+\frac{\delta s}{m}-1
\end{array}\right) .
$$


Thus, it suffices to verify that

$$
\left(\sum_{j=1}^{\delta-2} q^{-s j}\right)-q\left[\begin{array}{l}
m-1 \\
m-2
\end{array}\right]_{q}=-q^{s}
$$

which is straightforward.

Case V-IX: These are straightforward to prove.

Proposition 25. If $m d=n, m \geqslant 2$ and $\operatorname{gcd}(\ell, m)=1$, we have that $|\operatorname{CDP}(n, w)|_{q}$ evaluated at $q=\exp (2 \pi i \ell / m)$ is equal to $|\operatorname{CDP}(d, w)|$.

Proof. In Lemma 24, the first three cases cancel, so we know that $|\operatorname{CDP}(n, w)|_{q}$ evaluated at the root of unity is equal to the sum of the six remaining cases. After reordering, the sum of the cases is given by the expression

$$
\begin{aligned}
& w \sum_{\substack{s \in \mathbb{Z} \\
s \equiv_{m} 0}}\left(\begin{array}{c}
2 d-1 \\
d+\frac{\delta s}{m}
\end{array}\right)-\sum_{\substack{s \in \mathbb{Z} \\
\delta \equiv_{m} 0 \\
s \equiv_{m} 0}}\left(\begin{array}{c}
2 d-1 \\
d+\frac{\delta s}{m}-1
\end{array}\right)+\sum_{\substack{s \in \mathbb{Z} \\
s \equiv_{m} 0}}\left(\begin{array}{c}
2 d-1 \\
d+\frac{\delta s}{m}-1
\end{array}\right) \\
& -\sum_{\substack{s \in \mathbb{Z} \\
\delta \equiv_{m} 1}}\left(\begin{array}{c}
2 d-1 \\
d+\frac{\delta s-1}{m}-1
\end{array}\right)-\sum_{j=1}^{w-1} \sum_{\substack{s \in \mathbb{Z} \\
j+1+\delta s \equiv_{m} 0}}\left(\begin{array}{c}
2 d-1 \\
d+\frac{\delta s+j+1}{m}-1
\end{array}\right)-\sum_{\substack{s \in \mathbb{Z} \\
\delta s \equiv_{m} 1}}\left(\begin{array}{c}
2 d-1 \\
d+\frac{\delta s-1}{m}
\end{array}\right) .
\end{aligned}
$$

We note that

$$
\sum_{\substack{s \in \mathbb{Z} \\
\delta s \equiv m 1}}\left(\begin{array}{c}
2 d-1 \\
d+\frac{\delta s-1}{m}
\end{array}\right)=\sum_{\substack{s \in \mathbb{Z} \\
\delta s \equiv_{m} 1}}\left(\begin{array}{c}
2 d-1 \\
d+\frac{\delta(-s)+1}{m}-1
\end{array}\right)=\sum_{\substack{s \in \mathbb{Z} \\
\delta s+1 \equiv_{m} 0}}\left(\begin{array}{c}
2 d-1 \\
d+\frac{\delta s+1}{m}-1
\end{array}\right)
$$

so we can merge the fifth and sixth sum in (22), and shift the index in the fourth sum. Furthermore, the second and third sum can be rewritten, by adding and subtracting the third sum. We get

$$
\begin{gathered}
w \sum_{\substack{s \in \mathbb{Z} \\
s \equiv m 0}}\left(\begin{array}{c}
2 d-1 \\
d+\frac{\delta s}{m}
\end{array}\right)+2 \sum_{\substack{s \in \mathbb{Z} \\
s \equiv_{m} 0}}\left(\begin{array}{c}
2 d-1 \\
d+\frac{\delta s}{m}-1
\end{array}\right)-\sum_{\substack{s \in \mathbb{Z} \\
\delta s \equiv_{m} 0}}\left(\begin{array}{c}
2 d-1 \\
d+\frac{\delta s}{m}-1
\end{array}\right) \\
-\sum_{\substack{s \in \mathbb{Z} \\
\delta-1+\delta s \equiv_{m} 0}}\left(\begin{array}{c}
2 d-1 \\
d+\frac{\delta(s+1)-1}{m}
\end{array}\right)-\sum_{j=0}^{w-1} \sum_{\substack{s \in \mathbb{Z} \\
j+1+\delta s \equiv_{m} 0}}\left(\begin{array}{c}
2 d-1 \\
d+\frac{\delta s+j+1}{m}-1
\end{array}\right) .
\end{gathered}
$$

The last three terms can be merged, and we do some further simplifications to obtain

$$
w \sum_{t \in \mathbb{Z}}\left(\begin{array}{c}
2 d-1 \\
d+\delta t
\end{array}\right)+2 \sum_{t \in \mathbb{Z}}\left(\begin{array}{c}
2 d-1 \\
d+\delta t
\end{array}\right)-\sum_{j=0}^{w+1} \sum_{\substack{s \in \mathbb{Z} \\
j+1+\delta \equiv_{m} 0}}\left(\begin{array}{c}
2 d-1 \\
d+\frac{\delta s+j+1}{m}-1
\end{array}\right) .
$$


Finally, we note that

$$
\sum_{j=0}^{w+1} \sum_{\substack{s \in \mathbb{Z} \\
j+1+\delta s \equiv_{m} 0}}\left(\begin{array}{c}
2 d-1 \\
d+\frac{\delta s+j+1}{m}-1
\end{array}\right)=\sum_{r \in \mathbb{Z}}\left(\begin{array}{c}
2 d-1 \\
d+r
\end{array}\right)
$$

and thus we have equality with (18) in Lemma 21.

\section{The subset cyclic sieving phenomenon}

Recall that $\operatorname{CDP}(n, w)$ is a family of lattice paths $L$ of length $2 n$, ending with a north step. Note that such a lattice path $L$ is in $\operatorname{CDP}_{k}(n, w)$ if and only if the binary word of $L$ is invariant under cyclic shift by $2 k$ steps. However, the action of shifting the area sequence $k$ steps and shifting the underlying binary word $2 k$ steps are not equivalent the family $\operatorname{CDP}(n, w)$ is not closed under such a shifting of the binary word. This curious observation leads us to make the following definition.

Definition 26. Let $Y \subseteq X$ be a set of combinatorial objects and $C_{n}=\langle g\rangle$ be a cyclic group acting on $X$. Let $f(q) \in \mathbb{Z}[q]$ have non-negative coefficients, such that $f(1)=|Y|$. Then $\left(Y \subset X, C_{n}, f(q)\right)$ exhibits the subset cyclic sieving phenomenon if for every $k \in[n]$ we have

$$
f\left(\omega_{n}^{k}\right)=\left|\left\{y \in Y: g^{k} \cdot y=y\right\}\right| .
$$

We shall need the following theorem by Alexandersson and Amini.

Theorem 27 ([AA19, Thm. 2.7]). Let $f(q) \in \mathbb{Z}[q]$ take non-negative integer values at all $n^{\text {th }}$ roots of unity, and let $X$ be a set of cardinality $f(1)$. Define

$$
S_{k}:=\sum_{j \mid k} \mu(k / j) f\left(\omega_{n}^{j}\right) \text { whenever } k \mid n .
$$

If $S_{k} \geqslant 0$ for all $k$, then there is a cyclic group $C_{n}$ acting on $X$, such that $\left(X, C_{n}, f(q)\right)$ is a CSP-triple.

Note that the integers $S_{k}$ are exactly the number of elements in $X$ which are in a $C_{n}$-orbit of size $k$.

Proposition 28. If $\left(Y \subset X, C_{n}, f(q)\right)$ exhibits the subset-CSP, then there is a group action $\hat{C}_{n}$ on $Y$ such that $\left(Y, \hat{C}_{n}, f(q)\right)$ is a CSP-triple.

Proof. Let

$$
S_{k}:=\sum_{j \mid k} \mu(k / j) f\left(\omega_{n}^{j}\right) .
$$

Then $S_{k}$ is the number of elements in $X$ in a $C_{n}$-orbit of size $k$, so $S_{k} \geqslant 0$ for all $k \geqslant 1$. The fact that a group action $\hat{C}_{n}$ exists on $Y$ now follows from Theorem 27 . 


\subsection{Lattice paths with subset CSP}

We shall now provide an example of the subset-CSP on a family of lattice paths.

Let $\operatorname{AVL}(n, w)$ be the set of lattice paths from $(0,0)$ to $(n, n)$ that never touch the diagonals $\pm w$.

Proposition 29. The maj-count of $\operatorname{AVL}(n, w)$ is given by

$$
|\operatorname{AVL}(n, w)|_{q}=\sum_{s \in \mathbb{Z}} q^{2 s^{2} w+s w}\left(\left[\begin{array}{c}
2 n \\
n+2 s w
\end{array}\right]_{q}-\left[\begin{array}{c}
2 n \\
n+w+2 s w
\end{array}\right]_{q}\right) .
$$

Proof. The proof is analogous to the inclusion-exclusion argument in Corollary 20.

Notice that $\operatorname{AVL}(n, w)$ is a subset of $\operatorname{AVL}(n, n+1)$ - the set of all lattice paths from $(0,0)$ to $(n, n)$.

Theorem 30. Let $n, w \geqslant 1$ such that $\operatorname{gcd}(n, w)=1$. Let $C_{n}$ act on $\operatorname{AVL}(n, w)$ by letting the generator $\beta$ shift the binary word associated with the path two steps. Then

$$
\left(\operatorname{AVL}(n, w) \subset \operatorname{AVL}(n, n+1),\langle\beta\rangle,|\operatorname{AVL}(n, w)|_{q}\right)
$$

is a subset-CSP-triple.

Proof. We need to evaluate $|\operatorname{AVL}(n, w)|_{q}$ at $n^{\text {th }}$ roots of unity. Let $q=\exp (2 \pi i \ell / m)$, where $m \mid n$ and $\operatorname{gcd}(\ell, m)=1$, so that $q$ is a primitive $m^{\text {th }}$ root of unity. Note that it follows that $\operatorname{gcd}(w, m)=1$ as well, and we introduce $d=n / m$. Our goal is to show that $|\operatorname{AVL}(n, w)|_{q}$ evaluates to the number of paths in $\operatorname{AVL}(n, w)$ fixed under a shift of $2 d$ steps. It is clear that such paths are in bijection with $\operatorname{AVL}(d, w)$. There are two cases to consider.

Case $\boldsymbol{m}$ even. Using the $q$-Lucas theorem, Lemma 6 , we have that

$$
\sum_{s \in \mathbb{Z}} q^{2 s^{2} w+s w}\left[\begin{array}{c}
2 n \\
n+2 s w
\end{array}\right]_{q}=\sum_{s \in \mathbb{Z}} e^{\frac{2 \pi i \ell w s(2 s+1)}{m}}\left(\begin{array}{c}
2 d \\
d+\lfloor 2 s w / m\rfloor
\end{array}\right)\left[\begin{array}{c}
0 \\
\{2 s w\}_{m}
\end{array}\right]_{q} .
$$

Notice that the $q$-binomial is 0 unless $m$ divides $2 s$. Hence, by letting $t:=2 s / m$, we can rewrite the sum as

$$
\begin{aligned}
\sum_{t \in \mathbb{Z}} e^{\pi i \ell w t(t m+1)}\left(\begin{array}{c}
2 d \\
d+t w
\end{array}\right) & =\sum_{t \in \mathbb{Z}} e^{\pi i t}\left(\begin{array}{c}
2 d \\
d+t w
\end{array}\right) \\
& =\sum_{t \in \mathbb{Z}}\left(\begin{array}{c}
2 d \\
d+2 t w
\end{array}\right)-\sum_{t \in \mathbb{Z}}\left(\begin{array}{c}
2 d \\
d+w+2 t w
\end{array}\right)
\end{aligned}
$$

since $\ell, w$ and $t m+1$ are all odd if $m$ is even. In a similar fashion,

$$
\sum_{s \in \mathbb{Z}} q^{2 s^{2} w+s w}\left[\begin{array}{c}
2 n \\
n+w+2 s w
\end{array}\right]_{q}
$$


is equal to

$$
\sum_{s \in \mathbb{Z}} e^{\frac{2 \pi i \ell w s(2 s+1)}{m}}\left(\begin{array}{c}
2 d \\
d+\lfloor w(2 s+1) / m\rfloor
\end{array}\right)\left[\begin{array}{c}
0 \\
\{w(2 s+1)\}_{m}
\end{array}\right]_{q}
$$

but the last term is always zero, since $m$ does not divide $w(2 s+1)$

Case $\boldsymbol{m}$ odd. We consider (23), and see that the $q$-binomial expression vanishes unless $s$ it is a multiple of $m$. We let $t=s / m$ and obtain

$$
\sum_{s \in \mathbb{Z}}\left(\begin{array}{c}
2 d \\
d+2 t w
\end{array}\right)
$$

For the other term in (24), $2 s+1$ must be an odd multiple of $m$ in order for the $q$-binomial to be non-zero. Thus, $t=(2 s+1) / m$ is an integer and the expression simplifies to

$$
\sum_{s \in \mathbb{Z}}\left(\begin{array}{c}
2 d \\
d+w+2 t w
\end{array}\right)
$$

In conclusion, in both above cases, $|\operatorname{AVL}(n, w)|_{q}$ evaluates to the expression we have for $|\operatorname{AVL}(d, w)|$.

Problem 2. Find a natural cyclic action $\hat{C}_{n}$ on $\operatorname{AVL}(n, w)$ that makes

$$
\left(\operatorname{AVL}(n, w), \hat{C}_{n},|\operatorname{AVL}(n, w)|_{q}\right)
$$

into a CSP-triple.

\section{Möbius action on binary words}

\subsection{A new instance of cyclic sieving on binary words}

Let $\mathrm{BW}(n)$ denote the set of binary words of length $n$, and define an action $\eta$ on $\mathrm{BW}(n)$ as

$$
\eta:\left(b_{1}, b_{2}, \ldots, b_{n}\right) \mapsto\left(\hat{b}_{n-1}, \hat{b}_{n}, b_{1}, b_{2}, \ldots, b_{n-2}\right)
$$

where $\hat{b}_{i}:=1-b_{i}$. Note that the shift is indeed two steps, and that $\eta^{\circ n}(\mathbf{b})=\mathbf{b}$ for all words b of length $n$. For example,

$$
\eta(101)=\hat{0} \hat{1} 1=101, \quad \eta(110010)=011100 .
$$

We extend the notation so that $\hat{\mathbf{b}}:=\left(\hat{b}_{1}, \ldots, \hat{b}_{n}\right)$.

Lemma 31. The number of words in $\mathrm{BW}(n)$ fixed under $\eta^{\circ m}$ is given by $2^{d}$ if $\frac{n}{d}$ is odd and 0 otherwise, where $d=\operatorname{gcd}(m, n)$. 
Proof. Because $\eta$ generates a cyclic group of order $n$, the number of words fixed by $\eta^{\circ m}$ is the same as the number of words fixed by $\eta^{\circ d}$. Therefore it suffices to show that if $n=k d$, then $\eta^{o d}$ fixes $2^{d}$ elements if $k$ is odd, and 0 elements otherwise.

Let $\mathbf{b} \in \mathrm{BW}(n)$ and partition $\mathbf{b}$ into $k$ contiguous blocks of length $d$. Note that $\eta^{\circ d}$ maps block $i$ onto block $i+2(\bmod k)$. Suppose now $\mathbf{b}$ is fixed under $\eta^{\circ d}$ and consider the following cases.

Case $\boldsymbol{k}$ even. We have that blocks $1,3,5, \ldots, k-1$ must all be equal. However, block $k-1$ and block 1 must also be different, as $\eta^{\circ d}$ not only shifts bits $2 d$ steps to the right, but also flips all bits that wrap around. This is impossible, so there cannot be any fixed words in this case.

Case $\boldsymbol{k}$ odd. A similar argument as above shows that all odd-indexed blocks are equal, all even-indexed blocks are equal, and an even block is given by flipping all bits in an odd block. Hence, the entire word is determined by the first block. There are $2^{d}$ such possibilities, as there are $d$ bits in a block.

Lemma 32. For fixed $n$, all the expressions

$$
\text { (A) } \sum_{k=0}^{n} q^{\left(\begin{array}{c}
k \\
2
\end{array}\right)}\left[\begin{array}{l}
n \\
k
\end{array}\right]_{q} \quad(B) \quad \prod_{j=0}^{n-1}\left(1+q^{j}\right) \quad(C) \quad \sum_{\mathbf{b} \in \mathrm{BW}(n)} q^{\operatorname{maj}(\mathbf{b})+\operatorname{maj}(\hat{\mathbf{b}})}
$$

are equal.

Proof. Identity $(A)=(B)$. This is simply a consequence of the $q$-binomial theorem, (see [KC01, p. 14])

$$
\prod_{j=0}^{n-1}\left(1+x q^{j}\right)=\sum_{k=0}^{n} q^{\left(\begin{array}{c}
k \\
2
\end{array}\right)}\left[\begin{array}{l}
n \\
k
\end{array}\right]_{q} x^{k}
$$

Identity $(B)=(C)$. We do induction over $n$. The base case $n=1$ is easy. Now assume that the identity holds for $n-1$. Consider a binary word $\mathbf{b}$ of length $n-1$. We can either append 0 or 1 to make a word $\mathbf{b}^{\prime}$ of length $n$. If the last bit of $\mathbf{b}$ is equal to the appended bit,

$$
\operatorname{maj}(\mathbf{b})+\operatorname{maj}(\hat{\mathbf{b}})=\operatorname{maj}\left(\mathbf{b}^{\prime}\right)+\operatorname{maj}\left(\hat{\mathbf{b}}^{\prime}\right) \text {, }
$$

otherwise, the right-hand side is larger by $n-1$.

Proposition 33. Let $\eta$ act on the binary words $\mathrm{BW}(n)$ as before. Then

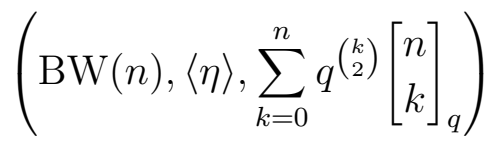

is a CSP-triple.

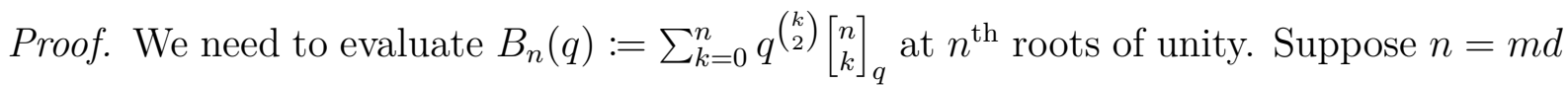
and $\xi=e^{2 \pi i \frac{\ell}{m}}$ with $\operatorname{gcd}(\ell, m)=1$. Note that Lemma 6 gives that

$$
\left[\begin{array}{l}
n \\
k
\end{array}\right]_{\xi}= \begin{cases}\left(\begin{array}{c}
d \\
k / m
\end{array}\right) & \text { if } m \mid k \\
0 & \text { otherwise. }\end{cases}
$$


Therefore,

$$
B_{n}(\xi)=\sum_{k=0}^{n} \xi^{\left(\begin{array}{c}
k \\
2
\end{array}\right)}\left[\begin{array}{l}
n \\
k
\end{array}\right]_{\xi}=\sum_{j=1}^{d} \xi^{\left(\begin{array}{c}
m j \\
2
\end{array}\right)}\left[\begin{array}{c}
n \\
m j
\end{array}\right]_{\xi}
$$

since only terms in the left hand side where $k$ is a multiple of $m$ contribute. We then get that

$$
B_{n}(\xi)=\sum_{j=0}^{d} e^{2 \pi i \frac{\ell}{m}\left(\begin{array}{c}
m j \\
2
\end{array}\right)}\left(\begin{array}{l}
d \\
j
\end{array}\right)=\sum_{j=0}^{d}(-1)^{\ell j(m j-1)}\left(\begin{array}{l}
d \\
j
\end{array}\right)= \begin{cases}0 & \text { if } m \text { even } \\
2^{d} & \text { otherwise }\end{cases}
$$

The two cases in the last step are as follows. If $m$ is even, then $\ell$ must be odd, and it follows that the sum is 0 . If $m$ is odd, then every term is positive and we get $2^{d}$.

That $\left(\mathrm{BW}(n),\langle\eta\rangle, \sum_{k=0}^{n} q^{\left(\begin{array}{c}k \\ 2\end{array}\right)\left[\begin{array}{l}n \\ k\end{array}\right]_{q}}\right)$ exhibits the CSP now follows from Lemma 31.

It would be of interest to find a representation-theoretic proof of Proposition 33. One possibility suggested by the referee is to use representation theory on the exterior algebra.

\subsection{Cyclic sieving on circular Möbius paths}

Recall the definition of circular Möbius paths, and Lemma 12, showing that $|\operatorname{CMP}(n)|=$ $2^{n-1}$. As $\operatorname{CMP}(n) \subseteq \operatorname{CDP}(n)$, we use the same definition of major index for circular Möbius paths as for the circular Dyck paths.

Let $\mathrm{OBW}(n) \subset \mathrm{BW}(n)$ be the set of binary words with odd parity. Note that the last bit can be deduced from the remaining word. We have a bijection $M: \operatorname{OBW}(n) \rightarrow$ $\operatorname{CMP}(n)$ by

$$
M\left(b_{1}, \ldots, b_{n}\right) \mapsto\left(b_{1}, b_{2}, \ldots, b_{n-1}, 0, \hat{b_{1}}, \hat{b_{2}}, \ldots, \hat{b}_{n-1}, 1\right) .
$$

Since $\eta$ preserves the parity of the word, $\eta$ act on $\mathrm{OBW}(n)$ and thus induces a $C_{n}$ action $\tilde{\eta}$ on $\operatorname{CMP}(n)$.

Example 34. Consider the first path of length 16 in Figure 4 . The first half of the corresponding binary word is given by 10110110, which is identified with $10110110 \in$ OBW $(n)$. We apply $\eta$ to this word and get 01101101 . We drop the last bit and append a 0: 01101100 this determines the first half of a new Möbius path in $\operatorname{CMP}(8)$, namely the second path in Figure 4.

Lemma 35. We have the following formula for $|\mathrm{CMP}(n)|_{q}, \bmod \left(q^{n}-1\right)$ :

$$
\sum_{\mathbf{b} \in \operatorname{CMP}(n)} q^{\operatorname{maj}(\mathbf{b})} \equiv \frac{1}{2} \sum_{k=0}^{n} q^{\left(\begin{array}{c}
k \\
2
\end{array}\right)}\left[\begin{array}{l}
n \\
k
\end{array}\right]_{q} \bmod \left(q^{n}-1\right) .
$$

Proof. First note that by the definition in (4),

$$
|\mathrm{CMP}(n)|_{q}=\sum_{\mathbf{b} \in \operatorname{CMP}(n)} q^{\operatorname{maj}(\mathbf{b})}=\sum_{\substack{\mathbf{b} \in \operatorname{BW}(n) \\ \mathbf{b}_{n}=0}} q^{\operatorname{maj}(\mathbf{b} \sim \hat{\mathbf{b}})}
$$


where $\sim$ denotes concatenation. Now observe that $\operatorname{maj}(\mathbf{b} \sim \hat{\mathbf{b}}) \equiv_{n} \operatorname{maj}(\mathbf{b})+\operatorname{maj}(\hat{\mathbf{b}})$. Any descent in the second half of the concatenation contributing to maj can simply be shifted by $n$. Furthermore, any contribution to maj by a descent between the first and second half must be exactly 0 or $n$. It is also clear by symmetry that

$$
\sum_{\substack{\mathbf{b} \in \mathrm{BW}(n) \\ \mathbf{b}_{n}=0}} q^{\operatorname{maj}(\mathbf{b} \sim \hat{\mathbf{b}})}=\sum_{\substack{\mathbf{b} \in \operatorname{BW}(n) \\ \mathbf{b}_{n}=1}} q^{\operatorname{maj}(\mathbf{b} \sim \hat{\mathbf{b}})} .
$$

This together with Lemma 32 implies the result.

We are now ready to present a CSP instance on circular Möbius paths of size $n$.

Theorem 36. The triples

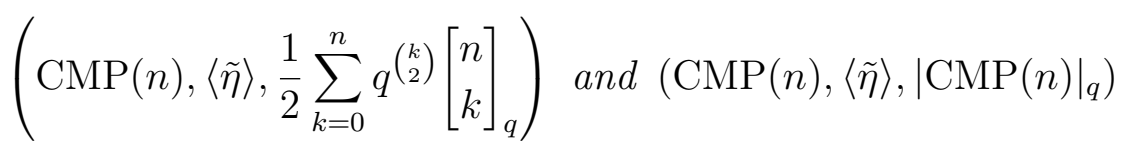

are CSP-triples, where $\langle\tilde{\eta}\rangle$ is the cyclic group of order $n$ that acts on $\operatorname{CMP}(n)$.

Proof. First, Lemma 35 implies that both CSP-instances are the same, up to a choice of a nice polynomial in $q$. The map $M$ above shows that the cyclic sieving phenomenon on $\operatorname{CMP}(n)$ is simply half of the cyclic sieving phenomenon in Proposition 33.

Remark 3. There is an alternative way to prove $\operatorname{CSP}$ on $\operatorname{CMP}(n)$. We view the elements in $\operatorname{CMP}(n)$ as $(\mathbf{x}, \mathbf{b})$, a starting point and a path with $2 n$ steps. Let $\beta$ act on the binary word $\mathbf{b}$ by cyclically shifting the path by two steps. Clearly, $\beta$ does not preserve the set $\operatorname{CMP}(n)$, but it is fairly easy to prove that

$$
\left(\operatorname{CMP}(n) \subset X,\langle\beta\rangle,|\operatorname{CMP}(n)|_{q}\right)
$$

is a subset-CSP-triple, where $X$ is chosen appropriately. Proposition 28 now implies $\operatorname{CMP}(n)$ with $|\operatorname{CMP}(n)|_{q}$ as the polynomial exhibits the CSP.

\section{$7 \quad$ Lyndon-like cyclic sieving}

Most results on cyclic sieving regard a family of combinatorial objects, where the cyclic group $C_{n}$ acts on a set $X_{n}$. The main result of this paper is no exception. In such cases, it is natural to pay extra attention to families where the various fixed points in $X_{n}$ under elements in $C_{n}$ are in bijection with $X_{k}$ for some $k \leqslant n$. This occurs when the group action is some type of cyclic shift on words, as we shall see. The notion of Lyndon-like cyclic sieving has implicitly been considered before from a different perspective. O. Gorodetsky [Gor19] introduced the notion of q-Gauss congruences for a family of polynomials, which turns out to be equivalent with (27) in the following definition. 
Definition 37. Let $\left\{\left(X_{n}, C_{n}, f_{n}(q)\right)\right\}_{n=1}^{\infty}$ be a family of instances of the cyclic sieving phenomenon. We say that the family is Lyndon-like if for every pair of positive integers $m$, $n$, with $m \mid n$, we have

$$
f_{n / m}(1)=f_{n}\left(e^{\frac{2 \pi i}{m}}\right) .
$$

By the definition of CSP, we have that

$$
f_{n}\left(e^{\frac{2 \pi i}{m}}\right)=\left|\left\{x \in X_{n}: g^{n / m}(x)=x\right\}\right|,
$$

where $\langle g\rangle=C_{n}$. Hence, the family is Lyndon-like if and only if the number of elements in $X_{n}$ fixed under $g^{d}$ is equal to $\left|X_{d}\right|$, for every $d \mid n$.

Apart from Theorem 22 and Theorem 30, there are several other Lyndon-like families of CSP instances. Here we list a few.

1. Words of length $n$, in the alphabet $[k]$, with $f_{n}(q)=\sum_{w \in[k]^{n}} q^{\operatorname{maj}(w)}$ as the polynomial.

2. A Lyndon-like CSP instance related to non-symmetric Macdonald polynomials is proved in [AU19]. The family $f_{n}(q)$ is defined as $f_{n}(q)=\sum_{T \in \operatorname{NAF}(n \lambda, k)} q^{\operatorname{maj}(T)}$, where $\operatorname{NAF}(\lambda, k)$ is a certain set of non-attacking fillings with maximal entry at most $k$ and shape $\lambda$. This CSP generalizes the Lyndon-like CSP on words.

Lemma 38. Let $\left\{\left(X_{n}, C_{n}, f_{n}(q)\right)\right\}_{n=1}^{\infty}$ be a Lyndon-like family of instances of the CSP. Then there are unique non-negative integers $\left\{t_{d}\right\}_{d=1}^{\infty}$ such that for every $n \geqslant 1$ we have

$$
\left|X_{n}\right|=\sum_{d \mid n} d \cdot t_{d}
$$

Proof. Let $O_{n, k}$ be the set of elements in $X_{n}$ that are in an orbit of size $k$ under $g$. We let $t_{n}:=\frac{1}{n}\left|O_{n, n}\right|$, so the identity we wish to prove is via Möbius inversion equivalent to the right-hand side of

$$
\left|X_{n}\right|=\sum_{d \mid n} d \cdot t_{d} \Longleftrightarrow\left|O_{n, n}\right|=\sum_{d \mid n} \mu\left(\frac{n}{d}\right)\left|X_{d}\right| .
$$

Now since the family is Lyndon-like, we have that for all $d \mid n$,

$$
\left|X_{d}\right|=\left|\left\{x \in X_{n}: g^{d}(x)=x\right\}\right|=\sum_{\substack{1 \leqslant k \leqslant d \\ k \mid d}}\left|O_{n, k}\right| .
$$

Thus, combining (28) and (29), it suffices to show that

$$
\left|O_{n, n}\right|=\sum_{\substack{1 \leqslant d \leqslant n \\ d \mid n}} \mu\left(\frac{n}{d}\right) \sum_{\substack{1 \leqslant k \leqslant d \\ k \mid d}}\left|O_{n, k}\right| .
$$


Möbius inversion on the outer sum gives that

$$
\left|X_{n}\right|=\sum_{\substack{1 \leqslant k \leqslant n \\ k \mid n}}\left|O_{n, k}\right|
$$

which is obviously true since every element in $X_{n}$ belongs to exactly one orbit of some size. The parameters $t_{n}$ are by construction unique.

We let the integers $t_{d}$ be called Lyndon parameters, since if we choose $t_{d}$ to be the usual Lyndon numbers, we get that $\left|X_{n}\right|=2^{n}$. A CSP instance with these parameters can be constructed by considering binary words of length $n$, with $C_{n}$ acting via cyclic shift. The Lyndon words of length $n$ are then in bijection with $\frac{1}{n}\left|O_{n, n}\right|$ in the notation above - that is, they are representatives of orbits of size $n$ under cyclic shift.

Lemma 39. There is no Lyndon-like family of CSP instances where $C_{n}$ acts on on some family of Catalan objects $\operatorname{Cat}(n)$.

Proof. We have that $|\operatorname{Cat}(1)|=1$ and $|\boldsymbol{C a t}(3)|=5$, so we must have $C_{3}$ acting on 5 objects. But then we must have $t_{1}=2, t_{3}=1$, or $t_{1}=5$, both of which are incompatible with $|\operatorname{Cat}(1)|=1$.

The goal of the remainder of this section is to prove the converse of Lemma 38. That is, there is a Lyndon-like family of CSP instances for any choice of Lyndon parameters.

Proposition 40. For any sequence of Lyndon parameters $T=\left\{t_{d}\right\}_{d=1}^{\infty}$, there is a Lyndonlike family of instances of the CSP.

Proof. It is enough to construct $X_{n}$ and a $C_{n}$-action on $X_{n}$ with the properties in Lemma 38 . Let

$$
X_{n}=\left\{(d, i, j): d \mid n, \quad 1 \leqslant i \leqslant t_{k}, \quad 1 \leqslant j \leqslant d\right\}
$$

and let the generator $g$ act as $g \cdot(d, i, j)=(d, i, j+n / d)$, where the last coordinate is taken modulo $d$. It is straightforward to show from the construction that for all $n$ and $k \mid n$

$$
\left|X_{n}\right|=\sum_{d \mid n} d \cdot t_{d} \quad \text { and } \quad\left|X_{k}\right|=\left|\left\{x \in X_{n}: g^{k}(x)=x\right\}\right| .
$$

We can then use $f_{n}(q)$ from Proposition 2 to make $\left(X_{n}, C_{n}, f_{n}(q)\right)$ into a CSP-triple.

It is possible to biject the $C_{n}$-action in Proposition 40 into an action on certain (however, quite artificial) words of length $n$, where $C_{n}$ acts by a one-step shift. Thus, it is no accident that the examples we have given above are all of this form.

Also note that the Lyndon parameters uniquely define the family of instances of the CSP in the following sense.

Proposition 41. Let $\left(X_{n}, C_{n}, f_{n}(q)\right)$ and $\left(Y_{n}, C_{n}, g_{n}(q)\right)$ be two families of Lyndon-like instances of the CSP with the same Lyndon parameters. Then there are $C_{n}$-equivariant bijections $\psi_{n}: X_{n} \rightarrow Y_{n}$ such that for all $g \in C_{n}$ and $x \in X_{n}, \psi_{n}(g \cdot x)=g \cdot \psi_{n}(x)$. 
Proof. First, we have that $\left|X_{n}\right|=\left|Y_{n}\right|$ for all $n$, by Lemma 38. From Definition 37, it then follows that

$$
\left\{x \in X_{n}: g^{n / k} \cdot x=x\right\}=\left|X_{n / k}\right|=\left|Y_{n / k}\right|=\left\{y \in Y_{n}: g^{n / k} \cdot y=y\right\} .
$$

By using Möbius inversion on (29), it is clear that the number of $C_{n}$-orbits of size $d$ in $X_{n}$ is equal to the number of $C_{n}$-orbits of size $d$ in $Y_{n}$. We can then simply let $\psi_{n}$ map orbits to orbits in an equivariant manner.

Remark 4. Note that Definition 37 gives a method to computationally check if a sequence of polynomials $\left\{f_{n}(q)\right\}_{n=1}^{\infty}$ might be completed to a Lyndon-like family of CSP instances. In this case, one might be able to narrow down the search for a suitable group action.

\section{Homomesy under area shift}

There is a concept called homomesy that means a statistic has the same average in each orbit as it has in the full space.

Let $\alpha^{\prime}$ denote the operation of shifting a binary sequence $\mathbf{b}$ ending in a 1 , cyclically so that the second to last 1 becomes the ending 1 . This corresponds to what happens with b when shifting the cyclic area sequence one step upwards.

Example 5. For $n=2$, we have the binary strings ending with a 1, partitioned into orbits by $\alpha^{\prime}:\{0011,1001\},\{0101\}$. The inversion numbers are $\operatorname{inv}(0011)=4, \operatorname{inv}(1001)=2$, $\operatorname{inv}(0101)=3$, so the average for each orbit is 3 . Note that the last orbit corresponds to two CDP with different starting point, but the inversion number is the same, so it is not important to keep track of here.

Theorem 42. For the words from circular Dyck paths, $\operatorname{inv}(w)$ is homomesic with respect to the action $\alpha^{\prime}$.

Proof. We only have to look at the orbit for any given word $\mathbf{b}$ that has $n 0 \mathrm{~s}, n$ 1s and ends in a 1. It turns out that it is not important here that it comes from a CDP. Let $z(\mathbf{b})=\left(z_{1}, \ldots, z_{n}\right)$ be the number of 0 s between two consecutive $1 \mathrm{~s}$ in $\mathbf{b}$, i.e. $z_{i}$ is the number of $0 \mathrm{~s}$ between the $(i-1)^{\text {th }}$ and $i^{\text {th }}$ one (with $z_{1}=$ number of leading $0 \mathrm{~s}$ ).

For example $\mathbf{b}=011001$ has $z(\mathbf{b})=(1,0,2)$. Then $\operatorname{inv}(\mathbf{b})=\sum_{i=1}^{n}(n-i+1) z_{i}$. The action $\alpha^{\prime}$ is rotating $z$ one step. Thus summing over an orbit of size $n$ we get

$$
\sum_{i} \sum_{j}(n-j+1) z_{i}=\sum_{i} z_{i}\left(\begin{array}{c}
n+1 \\
2
\end{array}\right)=n \cdot\left(\begin{array}{c}
n+1 \\
2
\end{array}\right) .
$$

Hence the average over the orbit is $\left(\begin{array}{c}n+1 \\ 2\end{array}\right)$.

If the orbit is of size strictly shorter than $n$ then the size still divides $n$, and we can sum over all $n$ shifts and the same calculation holds to compute the average.

The number of inversions is not homomesic with respect to the operation $\eta$ discussed in Section 6. 


\section{$9 \quad$ Further research directions}

Computer experiments suggests that the CSP instance in Theorem 22 can be refined by taking the number of circular peaks of the circular Dyck path into account. A circular peak of a (circular) Dyck path is an index $i \in[n]$ such that $a_{i+1} \leqslant a_{i}$ in its area sequence, where the index is taken $\bmod n$. In Example 9, 2, 4 and 6 are circular peaks. Whenever $\mathbf{a}$ is an ordinary Dyck path, with $a_{1}=0$, then the number of (ordinary) peaks of $\mathbf{a}$ is the same as the number of circular peaks.

Furthermore, one can also consider Schröder paths, where diagonal steps are allowed. We do not have an enumeration formula for these; it is an interesting open problem as that would count certain circular vertical-strip LLT polynomials, see [AP18].

One could also introduce $\operatorname{CMP}(n, w) \subseteq \operatorname{CDP}(n, w)$ as the set of circular area sequences satisfying the additional Möbius restriction in (4). Using the machinery above with some modification, it should be fairly easy to derive analogous expressions for enumeration.

\section{Acknowledgement}

P.A. is funded by the Knut and Alice Wallenberg Foundation (2013.03.07). S.L. and S.P. were supported by the Swedish Research Council grant 621-2014-4780.

\section{References}

[AA19] Per Alexandersson and Nima Amini. The cone of cyclic sieving phenomena. Discrete Mathematics, 342(6):1581-1601, 2019. doi:10.1016/j.disc.2019. 01.037.

[And76] George E. Andrews. The Theory of Partitions. Encyclopedia of Mathematics and its Applications. Cambridge University Press, 1976. doi:10.1017/ cbo9780511608650.

[AP18] Per Alexandersson and Greta Panova. LLT polynomials, chromatic quasisymmetric functions and graphs with cycles. Discrete Mathematics, 341(12):34533482, December 2018. doi:10.1016/j.disc.2018.09.001.

[AS18] Connor Ahlbach and Joshua P. Swanson. Refined cyclic sieving on words for the major index statistic. European Journal of Combinatorics, 73:37-60, October 2018. doi:10.1016/j.ejc.2018.05.003.

[AU19] Per Alexandersson and Joakim Uhlin. Cyclic sieving, skew Macdonald polynomials and Schur positivity. ArXiv e-prints, 2019. arXiv:1908.00083.

[BM12] Karin Baur and Volodymyr Mazorchuk. Combinatorial analogues of adnilpotent ideals for untwisted affine Lie algebras. Journal of Algebra, 372:85107, December 2012. doi:10.1016/j.jalgebra.2012.08.025.

[com08] The Sage-Combinat community. Sage-combinat: enhancing Sage as a toolbox for computer exploration in algebraic combinatorics. http://combinat. sagemath.org, 2008. 
[Dev19] The Sage Developers. Sagemath, the Sage Mathematics Software System Version 8.8. http://www. sagemath.org, 2019.

[Ell17a] Brittney Ellzey. Chromatic quasisymmetric functions of directed graphs. In 29th International Conference on Formal Power Series and Algebraic Combinatorics, volume 78B. Séminaire Lotharingien de Combinatoire, 2017. 12 pages. URL: https://www.emis.de/journals/SLC/wpapers/FPSAC2017/74\% 20Ellzey.pdf.

[Ell17b] Brittney Ellzey. A directed graph generalization of chromatic quasisymmetric functions. ArXiv e-prints, 2017. arXiv:1709.00454.

[FH85] Johannes Fürlinger and Josef Hofbauer. q-Catalan numbers. Journal of Combinatorial Theory, Series A, 40(2):248-264, November 1985. doi:10.1016/ 0097-3165(85) 90089-5.

[Gor19] Ofir Gorodetsky. q-congruences, with applications to supercongruences and the cyclic sieving phenomenon. International Journal of Number Theory, May 2019. doi:10.1142/s1793042119501069.

[Hag07] James Haglund. The q,t-Catalan numbers and the space of diagonal harmonics (University Lecture Series). American Mathematical Society, 2007. URL: https://www . math. upenn. edu/ jhaglund/books/qtcat.pdf.

[KC01] Victor Kac and Pokman Cheung. Quantum Calculus (Universitext). Springer, 2001. doi:10.1007/978-1-4613-0071-7.

[Moh79] Sri Gopal Mohanty. Lattice Path Counting and Applications. Probability and Mathematical Statistics. Academic Press, New York, 1979. doi:10.1016/ C2013-0-07442-7.

[Pec14] Oliver Pechenik. Cyclic sieving of increasing tableaux and small Schröder paths. Journal of Combinatorial Theory, Series A, 125:357-378, July 2014. doi:10. 1016/j.jcta.2014.04.002.

[PPR08] T. Kyle Petersen, Pavlo Pylyavskyy, and Brendon Rhoades. Promotion and cyclic sieving via webs. Journal of Algebraic Combinatorics, 30(1):19-41, September 2008. doi:10.1007/s10801-008-0150-3.

[Rho10] Brendon Rhoades. Cyclic sieving, promotion, and representation theory. Journal of Combinatorial Theory, Series A, 117(1):38-76, January 2010. doi: 10.1016/j.jcta.2009.03.017.

[RSW04] Victor Reiner, Dennis Stanton, and Dennis E. White. The cyclic sieving phenomenon. Journal of Combinatorial Theory, Series A, 108(1):17-50, October 2004. doi:10.1016/j.jcta.2004.04.009.

[Sag92] Bruce E. Sagan. Congruence properties of $q$-analogs. Advances in Mathematics, 95(1):127-143, September 1992. doi:10.1016/0001-8708(92) 90046-n.

[Sag11] Bruce E. Sagan. The cyclic sieving phenomenon: a survey. In Surveys in Combinatorics 2011, editor Robin Chapman, pages 183-234. Cambridge University Press, 2011. doi:10.1017/cbo9781139004114.006. 
[Slo19] Neil J. A. Sloane. The On-Line Encyclopedia of Integer Sequences. Online, 2019. URL: https://oeis.org.

[Sta11] Richard P. Stanley. Enumerative Combinatorics: Volume 1. Cambridge University Press, 2nd edition, 2011. doi:10.1017/CB09781139058520.

[Sta15] Richard P. Stanley. Catalan Numbers. Cambridge University Press, 2015. doi: $10.1017 /$ CB09781139871495.

[Ste94a] John R. Stembridge. On minuscule representations, plane partitions and involutions in complex Lie groups. Duke Mathematical Journal, 73(2):469-490, February 1994. doi:10.1215/s0012-7094-94-07320-1.

[Ste94b] John R. Stembridge. Some hidden relations involving the ten symmetry classes of plane partitions. Journal of Combinatorial Theory, Series A, 68(2):372-409, November 1994. doi:10.1016/0097-3165(94)90112-0.

[Ste96] John R. Stembridge. Canonical bases and self-evacuating tableaux. Duke Mathematical Journal, 82(3):585-606, March 1996. doi:10.1215/ s0012-7094-96-08224-1. 


\section{Corrigendum - Added November 6, 2020}

\section{Missing reference}

We have learned since the publication that the formula for the maj-generating polynomial for the paths we study was already known. Corollary 21 in our paper follows from Corollary 3 in [KM93]. The proof techniques are similar but somewhat different, using different maps. Given this result by Krattenthaler and Mohanty we could have omitted the five pages leading up to Corollary 21 in our paper. Also, our Proposition 30, which we proved with the same methods, is a special case of the result in [KM93].

We also want to correct the name we give to the $q$-Catalan numbers in Proposition 8. They are MacMahon's q-Catalan numbers.

\section{References}

[KM93] Christian Krattenthaler and Sri Gopal Mohanty. On lattice path counting by major index and descents. European Journal of Combinatorics, 14(1):43-51, January 1993. doi:10.1006/eujc.1993.1007.

\section{Erratum - Added November 18, 2021}

The original published version was not typeset correctly. Every time there was a reference to a Lemma X, Proposition X, Corollary X, etc it referred to Theorem X. This has now been corrected. No other changes were made. 